\title{
Primer registro de megafloras y palinología en estratos de la Formación Tarija (Pennsylvaniano), Arroyo Aguas Blancas, Provincia de Salta, Argentina. Descripción de dos especies nuevas
}

\author{
Mercedes di Pasquo \\ Consejo Nacional de Investigaciones Cientificas y Técnicas. Departamento de Geología, Facultad de Ciencias Exactas y Naturales, \\ Universidad de Buenos Aires. Ciudad Universitaria, Pabellón 2, $1^{\circ}$ Piso. (1428) Capital Federal. Argentina. \\ medipa@gl.fcen.uba.ar
}

RESUMEN. Se analiza e ilustra, por primera vez, una tafoflora hallada en estratos de la Formación Tarija que aflora en Arroyo Aguas Blancas, Provincia de Salta, Argentina. Se describen, también, dos especies nuevas: Malanzania starckii (licofita) y Grumosisporites delpapae (espora trilete). Se reconocieron improntas de semillas platispérmicas (Samaropsis nunezii García emend. A. Archangelsky, Cordaicarpus cesariae Gutiérrez, Ganuza, Morel y Arrondo emend. A. Archangelsky), improntas de tallos articulados (Paracalamites australis Rigby emend. Zampirolli y Bernardes de Oliveira) y compresiones/improntas de fragmentos foliares (Cordaites riojanus Archangelsky y Leguizamón, Ginkgophyllum sp. cf. G. diazii Archangelsky y Arrondo). Esta asociación es atribuida al Pennsylvaniano s.l. sobre la base de la distribución estratigráfica de las especies seminales. La asociación palinológica se compone de 101 especies, 53 de ellas reconocidas como autóctonas y 48 retrabajadas. El primer conjunto comprende 34 especies de esporas trilete, 11 granos de polen monosacado, un grano bisacado y siete especies de algas. El grupo retrabajado se compone de 27 esporas trilete y criptosporas, 20 especies de acritarcas, prasinofitas y otras algas y un quitinozoario. La presencia de especies exclusivas de la Biozona D. bireticulatus-C. chacoparanensis (BC) (e.g., Dictyotriletes bireticulatus (Ibrahim) Potonié y Kremp emend. Smith y Butterworth, Crucisaccites latisulcatus Lele y Maithy, Verrucosisporites morulatus (Knox) Potonié y Kremp emend. Smith y Butterworth), permite acotar su edad al Bashkiriano tardío-Moscoviano. La vegetación, compuesta principalmente por licofitas, esfenofitas, pteridofitas y gimnospermas, se habría desarrollado en diferentes ambientes continentales como lagos y ríos alimentados por glaciares de montaña.

Palabras claves: Megafloras, Malanzania starckiisp. nov., Palinofloras, Grumosisporites delpapaesp. nov., Cronología, Paleoambiente, Formación Tarija, Pennsylvaniano, Salta, Argentina. 


\begin{abstract}
First record of megafloras and palynology in the Tarija Formation (Pennsylvanian), Aguas Blancas creek, Salta Province, Argentina. Description of two new species. Fossil plant remains found in the Tarija Formation cropping out at the Aguas Blancas creek, Aguaragüe range, Salta Province, Argentina, are analysed and illustrated for the first time. Two new species, Malanzania starckii (Lycophyte) and Grumosisporites delpapae (trilete spore) are described. Molds and impressions of platispermic seeds (Samaropsis nunezii García emend. A. Archangelsky, Cordaicarpus cesariae Gutiérrez, Ganuza, Morel and Arrondo emend. A. Archangelsky), impressions of articulated stems (Paracalamites australis Rigby emend. Zampirolli and Bernardes de Oliveira) and compressions/impressions of fragmented leaves (Cordaites riojanus Archangelsky and Leguizamón, Ginkgophyllum sp. cf. G. diazii Archangelsky and Arrondo) were recorded. This assemblage is attributed to the Pennsylvanian s.l. based on stratigraphic ranges of platispermic seeds. The palynological assemblage is made up of 101 species of which 53 are authocthonous and 48 are reworked. The former group is composed of 34 trilete spore species, 11 monosaccate pollen grains, one bisaccate pollen grain and seven algal species. The reworked group comprises 27 trilete spores and cryptospores, 20 species of acritarchs, prasinophytes and other algae and one chitinozoan. The whole assemblage is attributed to the late BashkirianMoscovian based on the recognition of exclusive species of the D. bireticulatus-C. chacoparanensis (BC) Zone (e.g., Dictyotriletes bireticulatus (Ibrahim) Potonié and Kremp emend. Smith and Butterworth, Crucisaccites latisulcatus Lele and Maithy, Verrucosisporites morulatus (Knox) Potonié and Kremp emend. Smith and Butterworth). The vegetation, mainly composed of Lycophytes, Sphenophytes, Pteridophytes and Gymnosperms, would have developed in different continental palaeoenvironments like lakes and rivers fed by mountain glaciers.
\end{abstract}

Keywords: Megafloras, Malanzania starckii sp. nov., Palynofloras, Grumosisporites delpapae sp. nov., Chronology, Palaeoenvironment, Tarija Formation, Pennsylvanian, Salta, Argentina.

\section{Introducción}

En el norte de Argentina (sur de la cuenca Tarija) las sedimentitas del Pennsylvaniano se reúnen en los Grupos Macharetí y Mandiyutí y su edad está basada en datos palinológicos mientras que el PérmicoTriásico está representado por el Grupo Cuevo (Fig. texto $1 \mathrm{~A}$ y B), aunque todavía no se han reportado registros fosilíferos para esta región (véase di Pasquo, 2003). Para ampliar la información geológica y paleontológica de las unidades mencionadas se remite al lector a los recientes trabajos publicados por Starck y del Papa (2006) y di Pasquo (2007a), respectivamente. En esta contribución se describen dos especies nuevas correspondientes a una licofita (Malanzania starckii sp. nov.) y a una espora trilete (Grumosisporites delpapae sp. nov.), y se analizan e ilustran por primera vez otros restos paleoflorísticos que incluyen tallos de pteridofitas (licofitas y esfenofitas) y semillas platispérmicas y restos foliares de gimnospermas (cordaitales y ginkgoales). La sección litológica en la cual se hallaron los fósiles aquí estudiados corresponde a la porción media a superior de la Formación Tarija, presente en la quebrada Aguas Blancas, sierra de Aguaragüe, Provincia de Salta (Figs. texto 1B y 2). Está compuesta principalmente por potentes capas de diamictitas grises entre las que se intercalan delgados bancos de areniscas y pelitas. La nueva especie de licofita procede de un nivel de areniscas lenticulares presente en la parte media del perfil (Fig. 2A), la cual fue erróneamente referida a las esfenofitas por Starck y del Papa (2006). Hacia arriba, entre bancos de diamictitas se halla una intercalación de capas heterolíticas de areniscas de grano fino y pelitas en cuyos planos de estratificación se encontraron improntas y moldes de semillas platispérmicas junto con otros restos de tallos de esfenofitas y hojas de gimnospermas. A estos hallazgos se suman nuevos datos palinológicos obtenidos de seis niveles de pelitas y diamictitas de la sección estudiada. Se discute la importancia cronológica y paleoambiental de cada uno de los grupos de fósiles encontrados y se amplía el esquema de correlación del Grupo Macharetí propuesto por di Pasquo (2003) para el norte de Argentina.

\section{Materiales y Métodos}

Los restos de megafloras aquí estudiados fueron recolectados por varios colegas (incluida la autora) en diferentes campañas entre 1998 y 2000. El perfil está expuesto en el camino al Pozo Tuyunti (perforado por Pan American Energy S.A.) cuya entrada se ubica a $6,7 \mathrm{~km}$ al norte de la localidad de Aguaray desde la ruta Nacional No. 34 (Fig. texto 1B). Por camino de tierra a partir del $\mathrm{km} \mathrm{4,5}$, aproximadamente, se reconocen los afloramientos de la parte media de la Formación Tarija (Fig. 2A) compuestos principalmente de potentes capas de diamictitas grises, macizas y estratificadas que alternan con ban- 


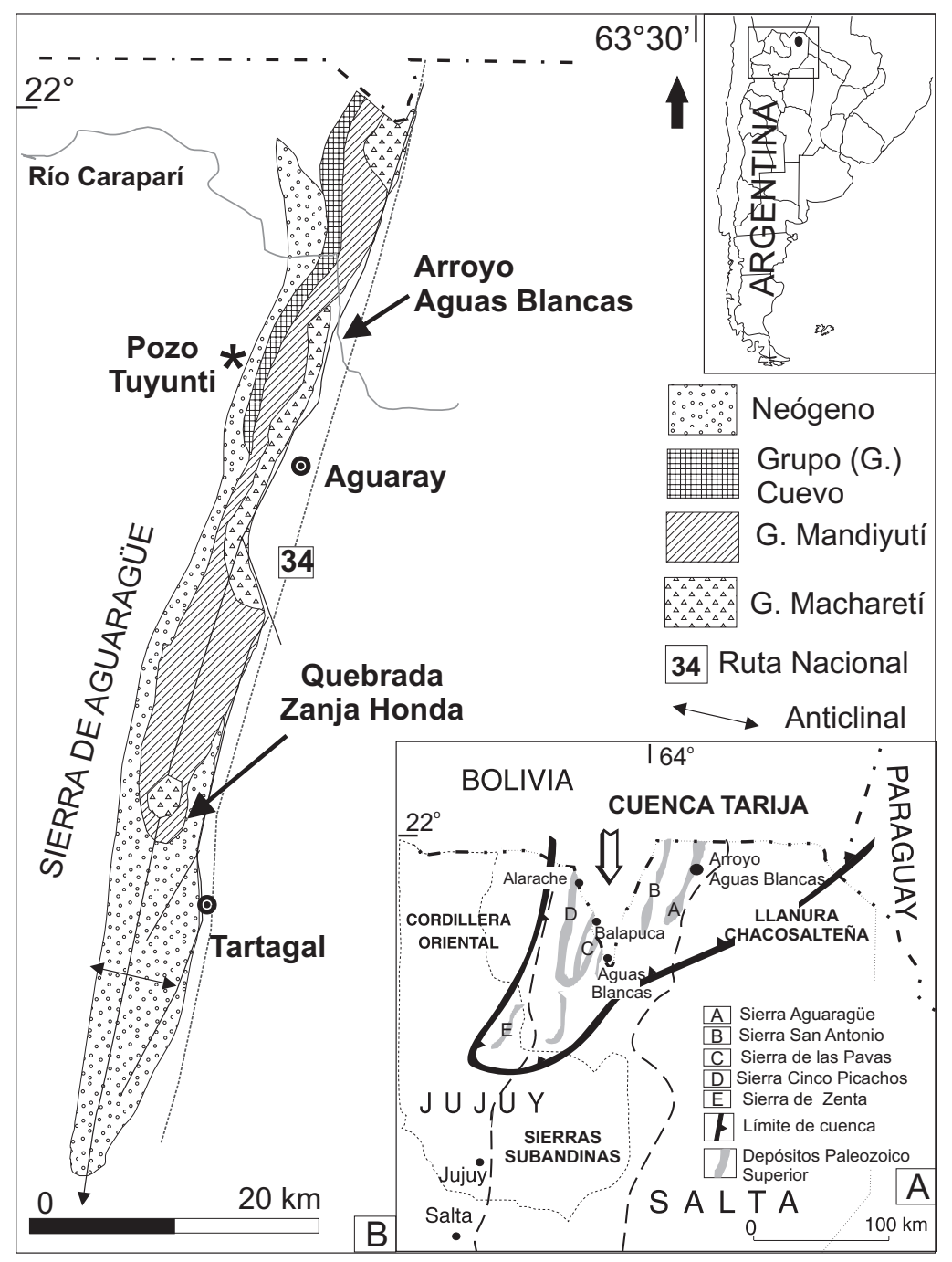

Fig. texto 1. A. Límite sur de la Cuenca Tarija donde se registran depósitos del Paleozoico Superior en el norte de Argentina y ubicación del área de estudio; B. Mapa geológico de las unidades del Paleozoico Superior en la sierra de Aguaragüe, norte de Salta, y localidades citadas en el texto (modificado de del Papa y di Pasquo, 2007).

cos lenticulares (en parte deformados) de areniscas rosadas de grano fino a medio con estructuras de corrientes (ondulitas y estratificación cruzada) o macizas principalmente en la parte inferior a media (Fig. texto 2A). En uno de estos bancos se encontraron restos fragmentarios de tallos de licofitas herbáceas dispuestos de forma desorientada como se ilustra en la figura texto 2B. Alrededor del $\mathrm{km} \mathrm{5,8}$ aparecen potentes bancos de diamictitas algo estratificadas color gris oscuro con restos carbonosos indeterminables de hojas y tallos fragmentados indeterminables (palinológicamente estéril) y hacia el techo se intercala un banco heterolítico compuesto por pelitas gris oscuro y areniscas blanquecino-grisáceas, de grano fino, en cuyos planos de estratificación se preservaron moldes, compresiones carbonosas e improntas de semillas platispérmicas, fragmentos foliares y tallos articulados (Fig. texto $2 \mathrm{~A} \mathrm{y} \mathrm{C}$ ). De un delgado banco $(10 \mathrm{~cm})$ de pelita gris oscuro en la base de dicha sección se obtuvo una asociación de palinomorfos (BAFC-Pl 1480). En niveles superiores de esta sección siguen otros bancos de diamictitas grises algo estratificadas, y en algunos casos se registraron briznas en su matriz pelítica. 


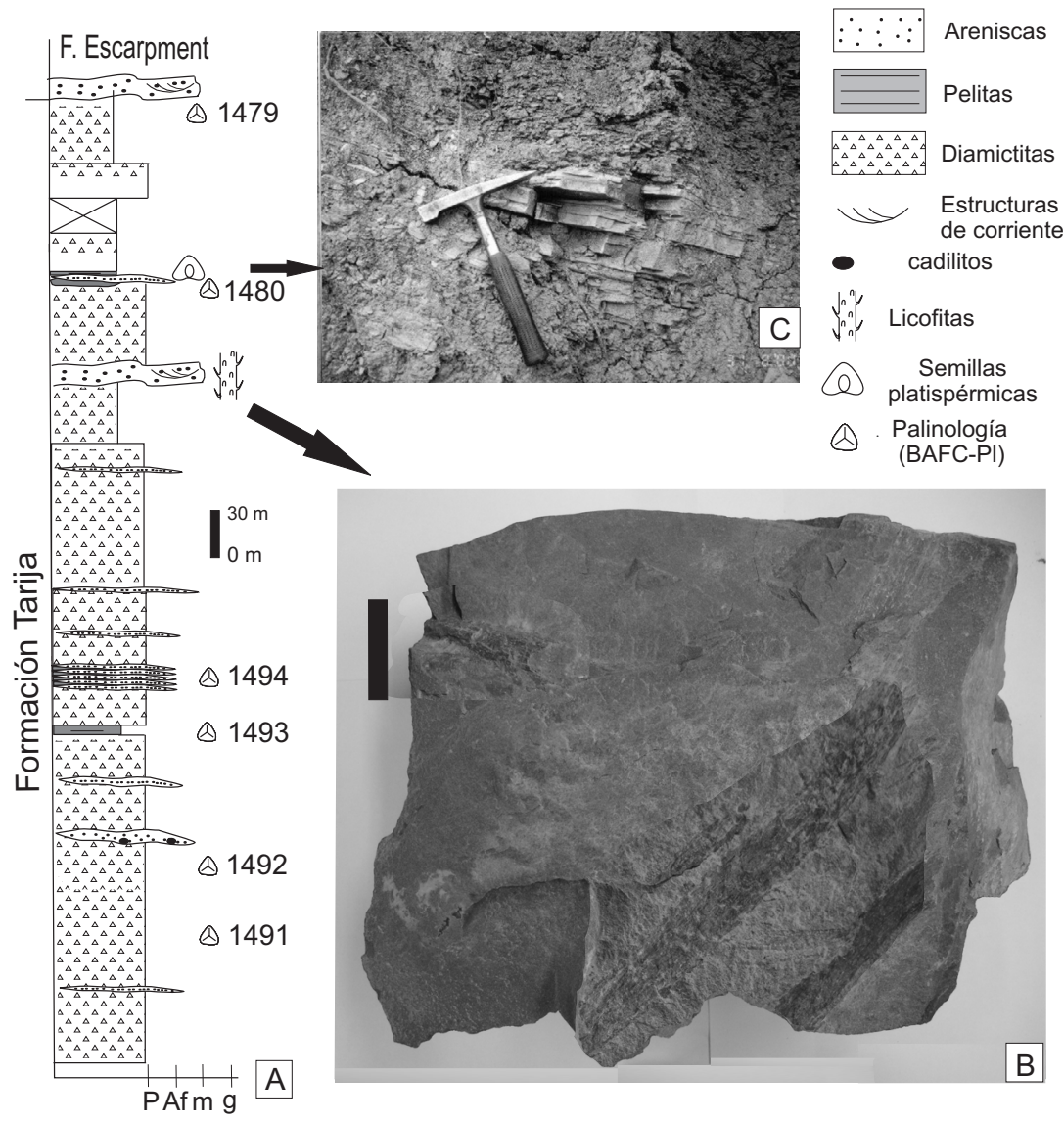

Fig. texto 2. A. Sección litoestratigráfica (modificada de del Papa y Martínez, 2001) de la Formación Tarija sobre el camino al pozo Tuyunti siguiendo en el arroyo Aguas Blancas y ubicación de las muestras paleontológicas aquí estudiadas; B. Bloque de arenisca de grano fino a medio (rosada) con restos de licofitas. BAFC-Pb 16368. Escala gráfica: $3,7 \mathrm{~cm}$; C. Capas de areniscas de grano fino y pelitas bien estratificadas sobre un banco de diamictita pobremente estratificada. En los planos de estratificación de las areniscas y pelitas se encuentran improntas y compresiones carbonosas de semillas platispérmicas y fragmentos foliares (BAFC-Pb 16355-16363). En niveles subyacentes, una pelita de color negro brindó palinomorfos (BAFCP1 1480). Escala gráfica (martillo geológico): $37 \mathrm{~cm}$ de longitud.

Un análisis sedimentológico más detallado de la Formación Tarija en esta localidad y otras en la sierra de Aguaragüe fue realizado por del Papa y Martínez (2001). Por su parte, Buatois y del Papa (2003) describieron trazas fósiles procedentes de la misma sección heterolítica mencionada arriba, de la cual se obtuvo además la muestra BAFC-Pl 1494 (Fig. texto 2A). Desafortunadamente, varias muestras resultaron palinológicamente estériles, entre ellas las areniscas de grano fino con licofitas, las pelitas del nivel con semillas platispérmicas, cuatro muestras de diferentes bancos de diamictita de la Formación Tarija y 12 muestras de pelitas castaño moradas y verdes recolectadas en las formaciones suprayacentes, Escarpment, San Telmo (Grupo
Mandiyutí), Cangapi y Vitiacua (Grupo Cuevo). Por lo tanto, las seis muestras fértiles aquí informadas corresponden a pelitas y diamictitas (véase la figura texto 2A) de color castaño, gris y gris verdoso claro, las cuales fueron procesadas siguiendo la metodología palinológica estándar. El residuo orgánico obtenido fue filtrado con malla de $25 \mu \mathrm{m}$ y fijado en preparados con gelatinaglicerina. Todas las muestras fueron rotuladas con la sigla BAFC-Pl, precediendo al número de preparado, correspondiente al repositorio (Palinoteca) ubicado en el Laboratorio de Palinoestratigrafía y Paleobotánica del Departamento de Geología de la Facultad de Ciencias Exactas y Naturales de la Universidad de Buenos Aires. Los palinomorfos y el grado de madurez de la materia 
orgánica fueron reconocidos mediante el uso de un microscopio binocular Orthoplan Leitz No. 4303017, con un aumento máximo de 1000x, y las coordenadas corresponden a la reglilla universal $\mathrm{En}$ gland Finder. Las microfotografías fueron obtenidas con cámaras digitales Pixera (1.2 Mpixel) y Motic (2.0 Mpixel) adosadas al microscopio y archivadas en formato de alta calidad (TIFF). Las muestras de megafloras (semillas y licofitas) fueron observadas en detalle a la lupa con un máximo de 50 aumentos e ilustradas con cámara digital Sony de resolución máxima 10 Mpixel y son alojadas en la colección de Paleobotánica de la institución citada bajo la sigla BAFC-Pb.

\section{Megafloras}

\subsection{Asociación de semillas platispérmicas, frag- mentos foliares y tallos (Fig. texto 2A y C, Lám. 1)}

Esta asociación se compone de moldes, compresiones carbonosas e improntas de semillas platispérmicas, restos foliares y tallos articulados (Lám. 1A), de regular preservación. Algunos de estos restos conservaron rasgos morfológicos suficientes para ser asignados a nivel específico. Entre ellos se reconocen fragmentos foliares atribuidos por su forma y tamaño a Ginkgophyllum sp. cf. G. diazii Archangelsky y Arrondo, 1974 (División Ginkgophyta) (Lám. 1F). Varios fragmentos son atribuidos a Cordaites riojanus Archangelsky y Leguizamón, 1980 (División Coniferophyta, Orden Cordaitales) por la forma del ápice y la venación paralela (Lám. 1G-J). Otro fragmento corresponde a un tallo articulado asignado a Paracalamites australis Rigby, 1966 emend. Zampirolli y Bernardes de Oliveira, 2000 (División Sphenophyta, Orden Equisetales) por su morfología de nodos e internodos con costulación continua (Lám. 1K). Este material muestra una gran similitud con el ilustrado por Zampirolli y Bernardes de Oliveira (2000) registrado en el Pennsylvaniano de la cuenca Paraná. Completan la asociación dos especies de semillas platispérmicas (División Coniferophyta, Orden Cardiocarpales Seward) asignadas a Samaropsis nunezii García, 1990 emend. Archangelsky, 2000 (Lám. 1A, D y E) y Cordaicarpus cesariae Gutiérrez, Ganuza, Morel y Arrondo, 1992 emend. Archangelsky, 2000 (Lám. 1B y C). Para su identificación se utilizaron principalmente los trabajos de Gutiérrez et al. (1992) y Archangelsky (2000).

\subsection{Asociación de licofitas (Fig. texto 2A, B, Lám. 2)}

Numerosas improntas y moldes de tallos de una única especie de licofita de porte herbáceo fueron recolectadas de un estrato lenticular compuesto por areniscas rosadas de grano fino.

\section{Descripciones sistemáticas}

\section{División Lycophyta \\ Género Malanzania Archangelsky, Azcuy y Wagner, 1981}

Especie tipo: Malanzania nana Archangelsky, Azcuy y Wagner, 1981. Pennsylvaniano, Provincia de La Rioja, Argentina.

\section{Malanzania starckii sp. nov.}

Fig. texto 2B, Lám. 2A-I

Holotipo: BACF-Pb 16369, Lám. 2E, G.

Paratipos: BAFC-Pb 16375, Lám. 2A, B; BAFCPb 16378, Lám. 2C; BAFC-Pb 16376, Lám. 2D; BAFC-Pb 16380, Lám. 2F; BAFC-Pb 16368, Lám. $2 \mathrm{H}, \mathrm{I}$.

Localidad tipo: Quebrada Aguas Blancas, Sierra de Aguaragüe, Provincia de Salta, Argentina.

Diagnosis: stems of uniform width, covered by 4 to 6 leaf bases (mamelons) per half gyre, irregularly oval in vertical, lepidodendroid phyllotaxis arranged showing a variable angle between $35^{\circ}\left(45^{\circ}\right) 50^{\circ}$ measured respect to the vertical. One spiniform, simple apex microphyll emerges from the medium to the upper part of each leaf base. Surface area between leaf bases smooth.

Dimensiones (42 ejemplares, BAFC-Pb 16364 a 16394): ancho de los tallos 1,0-2,0 cm, bases foliares de 3-4 $\mathrm{mm}$ de alto por 2-3 $\mathrm{mm}$ de ancho, separación entre bf 2-4 mm (medida en diagonal), micrófilas 4-7 $\mathrm{mm}$ de largo por 1,5-2,5 $\mathrm{mm}$ de ancho en la base. Longitud máxima de los tallos $17 \mathrm{~cm}$ (corresponde al ejemplar ilustrado en la figura texto 2B y Lám. 2, H, I).

Descripción del holotipo (BAFC-Pb 16369, Lám. 2E y G): ejemplar de tallo ('cast') compuesto por numerosas bases foliares con micrófilas visibles principalmente en ambos márgenes. Las bases foliares, de 5 a 6 por medio giro, presentan forma irregularmente oval, $c a .3 \mathrm{~mm}$ de diámetro mayor en sentido vertical, y se disponen de forma helicoidal lepidodendroide formando un ángulo de $35^{\circ}$, 
aproximadamente, medido con respecto a la vertical; se encuentran distanciadas entre sí de 3 a $4 \mathrm{~mm}$ medido en diagonal. En la parte media a superior de cada base foliar se inserta una micrófila espiniforme de ápice simple, de 4 a $5 \mathrm{~mm}$ de largo máximo, levemente curvado hacia arriba. Sobre el tallo, la presencia de las micrófilas produce una distorsión de la forma de las bases foliares. La superficie del tallo entre las bases foliares es lisa.

Derivatio nominis: dedicado al Licenciado Daniel Starck, destacado geólogo que encontró el nivel de licofitas.

Comentarios: los tallos se preservaron como cast, moldes externos e improntas con diferentes niveles de decorticación y de manera parcial algunos ejemplares aún conservan una película de carbón (Lám. $2 F$ ). Por ello, se observan variaciones tanto en la forma de las bases foliares como en la presencia de manera completa o incompleta de micrófilas (falsas cicatrices foliares?) a lo largo de un tallo, relacionado con el nivel de decorticación de los distintos especímenes estudiados.

Comparaciones: Malanzania nana Archangelsky et al., 1981, del Pennsylvaniano de La Rioja, se diferencia por presentar falsas cicatrices foliares de menor tamaño (0,5 a $1 \mathrm{~mm})$, de forma subcircular a subrómbicas en sentido horizontal y más separadas entre sí con relación al tamaño de las cicatrices; la disposición lepidodendroide presenta un ángulo mayor de alrededor de $60^{\circ}$ y las hojas espiniformes son de menor tamaño (1 mm de largo). Malanzania ottonei Carrizo y Azcuy, 1998, del Mississippiano de La Rioja, difiere principalmente por su tamaño bastante menor (el ancho máximo de los tallos es de $5 \mathrm{~mm}$ ), forma subcircular a subtriangular de las falsas cicatrices foliares en sentido horizontal, una mayor separación entre ellas (una y media veces a tres veces y media su diámetro), y micrófilas de menor tamaño (1,5 $\mathrm{mm}$ de largo).

\section{Palinología}

Se describe en primer lugar una nueva especie de espora y luego se dan las características de la asociación palinológica. En la figura texto 4A y B se presenta la lista de especies autóctonas y retrabajadas, con su autoría completa, su distribución cuantitativa en las muestras estudiadas y la ubicación de las formas ilustradas en las láminas 3 a 6 . Asimismo, se incluye una columna donde se cita una selección de trabajos sobre la distribución estratigráfica de todas las especies, las cuales son ampliamente conocidas en la literatura palinológica de Argentina y del resto del mundo, por lo que no se precisa detallar su descripción morfológica. Los especímenes ilustrados en la lámina $3 \mathrm{H}, \mathrm{O}$ y R presentan una preservación regular, pero conservan los rasgos morfológicos necesarios para sostener su asignación específica.

\subsection{Descripciones sistemáticas}

Anteturma Proximegerminantes Potonié, 1970

Turma Triletes Reinsch emend. Dettmann, 1963

Suprasubturma Laminatitriletes

Smith y Butterworth, 1967

Subturma Azonolaminatitriletes

Smith y Butterworth, 1967

Infraturma Tuberculornati

Smith y Butterworth, 1967

Género Grumosisporites

Smith y Butterworth, 1967

Especie tipo: Grumosisporites verrucosus (Butterworth y Williams) Smith y Butterworth, 1967. Serpukhoviano de Escocia.

Afinidad botánica: Pteridophyta (Balme, 1995).

\section{Grumosisporites delpapae sp. nov.}

$$
\text { (Lám. 3. M, N) }
$$

Holotipo: BAFC-Pl 1494(2) P37/4 (Lám. 3.N). Paratipo: BAFC-Pl 1491(1) D27 (Lám. 3.M). Localidad tipo: Quebrada Aguas Blancas, Sierra de Aguaragüe, Provincia de Salta, Argentina.

Diagnosis: spore radial, trilete, camerate, amb circular to sub-circular. Laesura simple, straight to somewhat sinuous, extending $3 / 4$ to $4 / 5$ of the spore radius. Intexine thin, circular to subcircular amb; slightly off-center, separated from the exoexine between $1 / 6$ and $3 / 4$ of the spore radius. Exoexine ornamented mostly with discrete subcircular to oval warts in plant, and hemispherical with round-topped to more rectangular with flat-topped in profile, 2 (3-5) $8 \mu \mathrm{m}$ in basal diameter and 1-3 $\mu \mathrm{m}$ in height; spaced at least $2 \mu \mathrm{m}$ or more appart. Around 30 warts are projected in the margin.

Dimensiones ( 9 especímenes): diámetro ecuatorial 40-50 $\mu \mathrm{m}$.

Derivatio nominis: dedicado a la Dra. Cecilia del Papa, quien contribuyó aportando algunas de las muestras aquí estudiadas. 
Comparaciones: esta especie se incluye en el género Grumosisporites por ser camerada y portar ornamentación verrucosa. La forma, tamaño y grado de fusión de las verrugas y su densidad son los principales rasgos que permiten separar las diferentes especies de este género, descritas por Smith y Butterworth (1967). Entre ellas, la especie tipo es la forma más próxima a la nueva especie, la cual se distingue por presentar una ornamentación de verrugas mayormente fusionada, formando costillas bajas separadas por canales meandriformes poco profundos. Otra forma camerada semejante es Spelaeotriletes sp. (in Azcuy y di Pasquo, 2005, pl. VI, 7, 8), la cual se diferencia por su ornamentación más variada de verrugas, conos y báculas.

\subsection{Características de la asociación palinológica}

La asociación palinológica en su conjunto se compone de 101 especies, 53 de ellas reconocidas como autóctonas, y 48 retrabajadas. La distribución estratigráfica conocida de las especies determinadas (Fig. texto 3) permitió identificar un conjunto de especies autóctonas, compuesto por 34 especies de esporas trilete, 11 granos de polen monosacados, un grano bisacado y siete especies de algas. El conjunto de especies retrabajadas contiene 27 especies de esporas y criptosporas y 21 de microplancton (Fig. texto 4A y B). Como ejemplo de distribución porcentual, el nivel BAFC-Pl 1492 (Fig. texto 2A) presenta un $40 \%$ de palinomorfos mientras que el $60 \%$ son fitoclastos (charcoal, leños, cutículas y fragmentos indeterminables). Entre los palinomorfos, el $63 \%$ corresponde al conjunto de especies autóctonas y el $30 \%$ a las retrabajadas, en tanto un $7 \%$ se agruparon como esporas indeterminables. A su vez, dentro del grupo autóctono, el $32 \%$ son granos de polen monosacado, $18 \%$ son esporas trilete representadas por $11 \%$ de pteridofitas, $4 \%$ de licofitas, $3 \%$ de esfenofitas, $12 \%$ de algas clorofíceas y el resto de esporas de pteridofitas/pteridospermafitas. El grupo retrabajado presenta un 13\% de esporas trilete/criptosporas y un $17 \%$ de acritarcas/prasinofitas y un ejemplar de quitinozoario. Una selección de palinomorfos autóctonos y retrabajados así como algunos restos de traqueidas y cutículas son ilustrados en las láminas 3 a 6 (véase la figura texto 4A y B). El color de los palinomorfos y fitoclastos es variable según los niveles; en general las esporas, granos de polen y fitoclastos se observan de color castaño claro

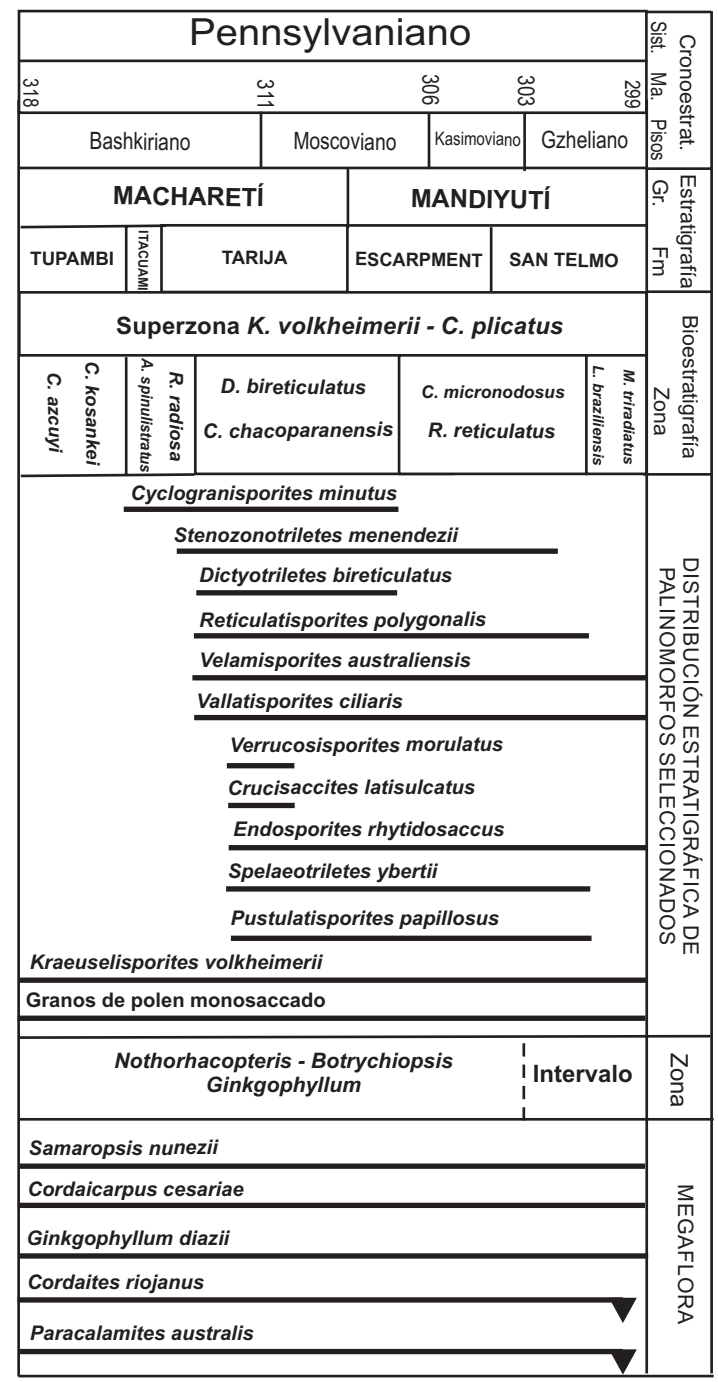

Fig. texto 3. Especies palinológicas seleccionadas por su distribución estratigráfica restringida, según el esquema de biozonación de los Grupos Macharetí y Mandiyutí propuesto por di Pasquo (2003) y la información provista por del Papa y di Pasquo (2007). La biozonación megaflorística y la distribución estratigráfica de las especies determinadas están tomadas principalmente de Gutiérrez (1995), Archangelsky et al. (1996), Archangelsky (1999) y Azcuy et al. (2007). Cronoestratigrafía y cronología absoluta tomada de Davydov et al. (2004).

a oscuro (TAI 2 a -3) y en algunos casos hasta casi negro (en especial algunas esporas retrabajadas, TAI $3+\mathrm{a}-4$ ), en tanto que Botryococcus y el resto de las algas autóctonas como también algunas especies de acritarcas y prasinofitas retrabajadas, presentan un 


\section{A. DISTRIBUCIÓN ESTRATIGRÁFICA Y CUANTITATIVA (NÚMERO DE EJEMPLARES) DE LAS ESPECIES AUTÓC TONAS Y RETRABAJADAS DETERMINADAS, CON SU AUTORÍA COMPLETA.}

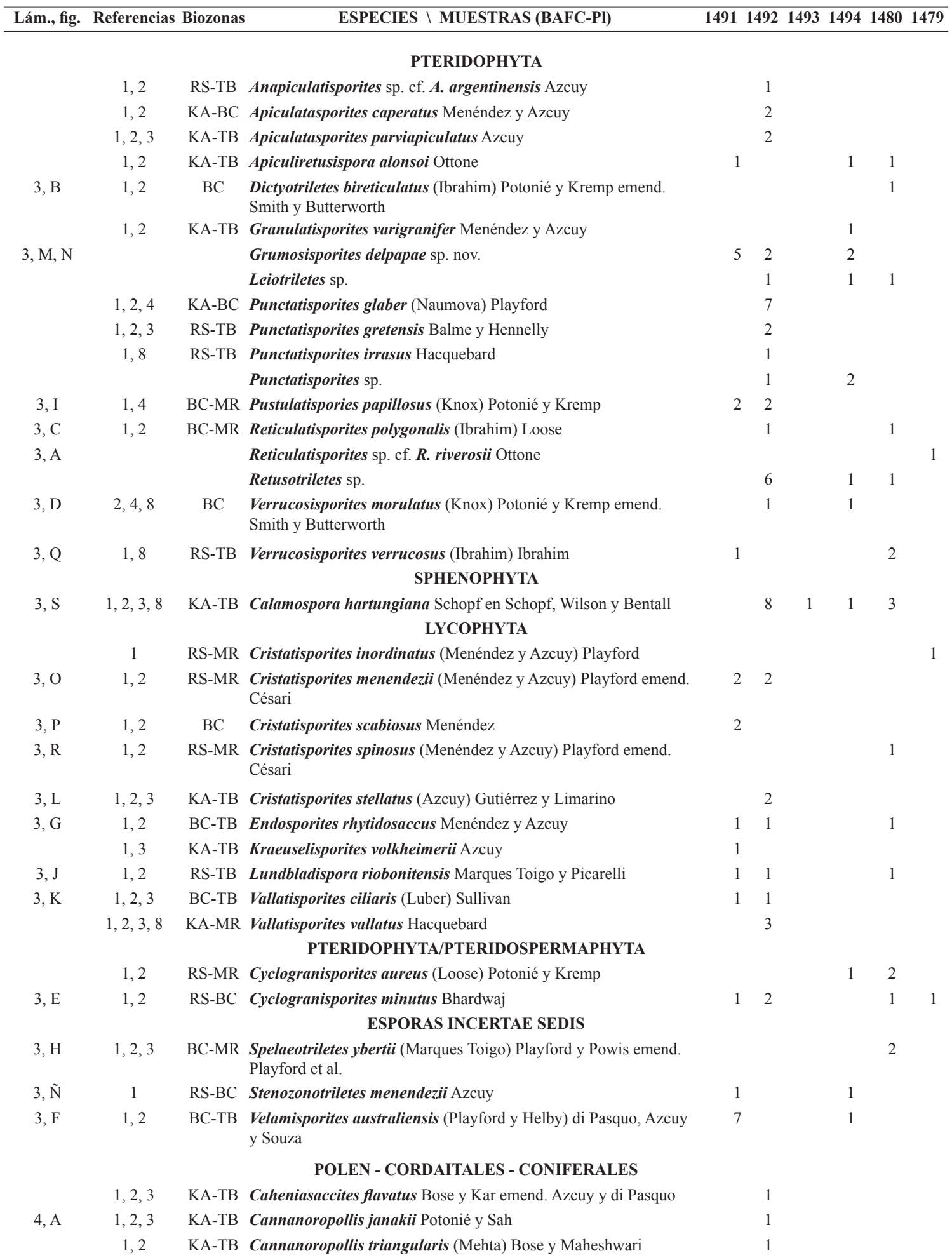


continuación Fig. Texto 4A.

\begin{tabular}{|c|c|c|c|c|c|c|c|c|c|}
\hline Lám., fig. & Referencias & Biozonas & ESPECIES \MUESTRAS (BAFC-PI) & 1491 & 1492 & 1493 & 1494 & 1480 & $0 \mathbf{1 4 7 9}$ \\
\hline \multirow[t]{2}{*}{$4, \mathrm{~B}$} & 1,2 & $\mathrm{BC}$ & Crucisaccites latisulcatus Lele y Maithy & & 1 & 1 & & & \\
\hline & 1,2 & RS-TB & Divarisaccus stringoplicatus Ottone & & 1 & 1 & & & \\
\hline \multirow[t]{2}{*}{$4, \mathrm{C}$} & $1,2,3$ & KA-TB & Plicatipollenites malabarensis (Potonié y Sah) Foster & 1 & 15 & & & & \\
\hline & $1,2,3$ & KA-TB & Potonieisporites barrelis Tiwari & & 6 & 6 & & & \\
\hline $4, \mathrm{E}$ & $1,2,3$ & KA-TB & Potonieisporites congoensis Bose y Maheshwari & & 3 & 3 & & & \\
\hline $4, \mathrm{D}$ & $1,2,3$ & KA-TB & Potonieisporites neglectus Potonié y Lele & & 2 & 2 & & & \\
\hline \multirow[t]{5}{*}{$4, \mathrm{G}$} & $1,2,3$ & KA-TB & Potonieisporites novicus Bhardwaj emend. Poort y Veld & & 22 & 2 & & & \\
\hline & $1,2,3$ & KA-TB & Potonieisporites triangulatus Tiwari & & 1 & 1 & & & \\
\hline & & & Monosacados fragmentados y/o sin cuerpo central & & 20 & 0 & & 3 & \\
\hline & & KA-TB & Monosacados spp. & & 10 & & & & \\
\hline & & & POLEN - PTERIDOSPERMAPHYTA & & & & & & \\
\hline \multirow[t]{2}{*}{$4, \mathrm{I}$} & 9 & & Pteruchipollenites gracilis (Segroves) Foster & & & & & & 1 \\
\hline & & & ALGAS & & & & & & \\
\hline 4, $\mathrm{H}$ & 1 & $\mathrm{BC}$ & Cymatiosphaera gondwanensis (Tiwari) Backhouse & & & & & & 1 \\
\hline $4, \mathrm{~F}, \mathrm{~J}, \mathrm{~L}-\mathrm{N}$ & $1,2,3,6$ & KA-TB & Botryococcus braunii Kützing & 4 & 20 & 15 & 15 & & 1 \\
\hline $4, \mathrm{~S}$ & $1,2,3$ & KA-TB & Brazilea scissa (Balme y Hennelly) Foster & & 6 & 6 & & & \\
\hline \multirow[t]{2}{*}{$4, \mathrm{U}$} & 1,2 & KA-TB & Quadrisporites horridus Hennelly ex Potonié y Lele & 1 & 3 & 3 & & & \\
\hline & 1,2 & RS-TB & Tetraporina spp. & 1 & 1 & 1 & & & \\
\hline
\end{tabular}

Fig. texto 4 A. Especies autóctonas agrupadas de acuerdo con sus afinidades botánicas más probables (véase Balme, 1995) en orden alfabético. En la tercera columna se consigna la distribución estratigráfica de cada especie correspondiente al esquema bioestratigráfico de los Grupos Macharetí y Mandiyutí (Pennsylvaniano de la Cuenca Tarija), publicado por di Pasquo (2003, véase Fig. texto 3) y los nuevos datos palinoestratigráficos presentados por del Papa y di Pasquo (2007).

\section{B. DISTRIBUCIÓN ESTRATIGRÁFICA Y CUANTITATIVA (NÚMERO DE EJEMPLARES) DE LAS ESPECIES AUTÓC- TONAS Y RETRABAJADAS DETERMINADAS, CON SU AUTORÍA COMPLETA.}

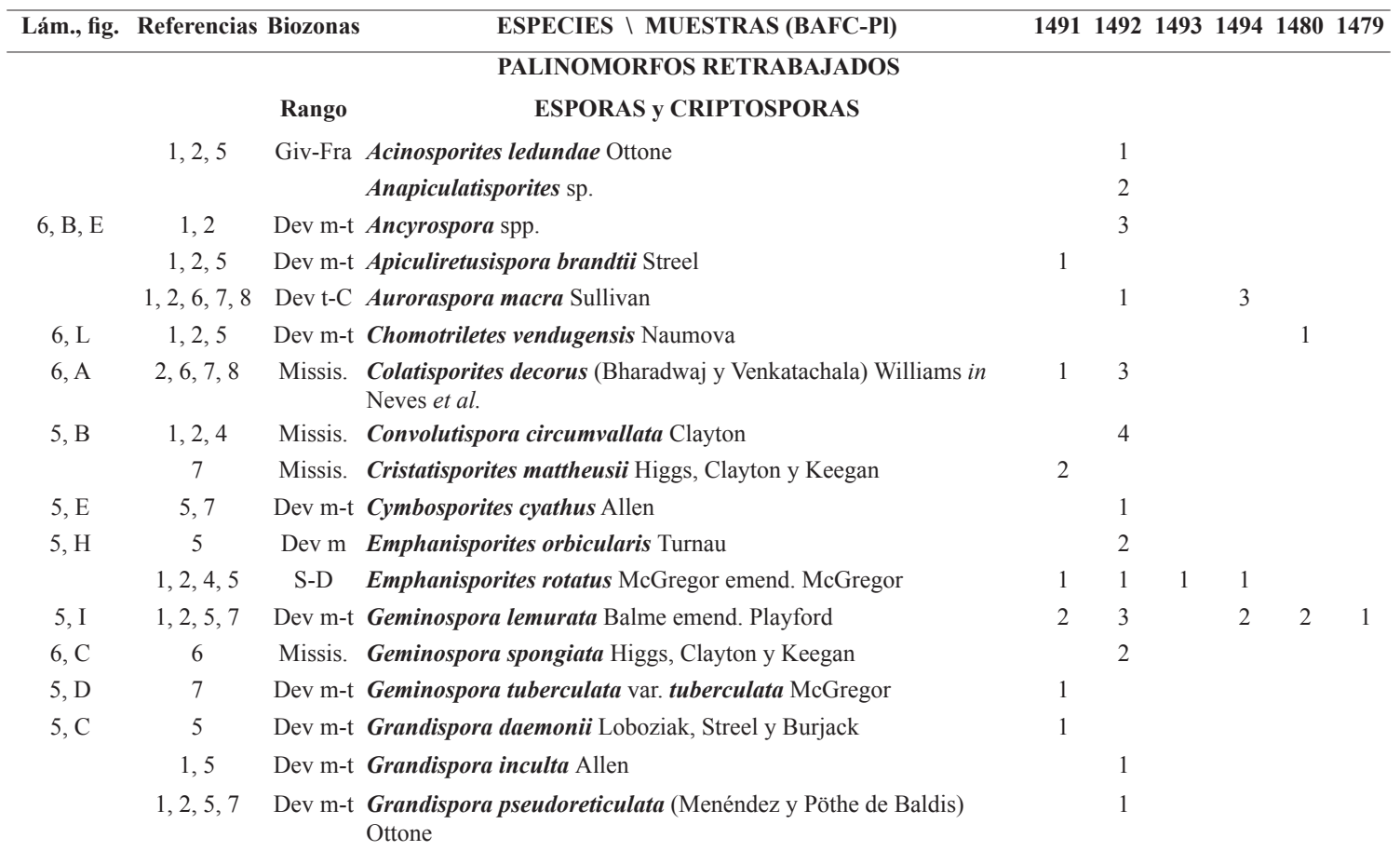


continuación Fig. Texto 4B.

\begin{tabular}{|c|c|c|c|c|c|c|c|c|c|}
\hline Lám., fig. & Referencias & Biozonas & ESPECIES \MUESTRAS (BAFC-PI) & 1491 & 1492 & 1493 & 1494 & 1480 & 1479 \\
\hline \multirow[t]{2}{*}{$5, \mathrm{G}$} & $1,2,6$ & $\begin{array}{l}\text { Dev t- } \\
\text { Missis. }\end{array}$ & Kraeuselisporites explanatus (Luber) Azcuy y di Pasquo & & & & 1 & & \\
\hline & 2,5 & S-D & Laeovolancis divellomedia (Chibrikova) Burgess y Richardson & 1 & 1 & & & & \\
\hline $6, \mathrm{D}$ & 4,8 & Missis. & Microreticulatisporites parvirugosus Staplin & 2 & & & 1 & & \\
\hline \multirow[t]{2}{*}{$5, \mathrm{~A}$} & $1,2,8$ & Dev s & Retispora lepidophyta (Kedo) Playford & 1 & 5 & & & & \\
\hline & & Dev m & Rhabdosporites parvulus Richardson & 1 & & & & & \\
\hline \multirow[t]{3}{*}{$5, \mathrm{~F}$} & 8 & Missis. & Velamisporites minutus (Neves y Ioannides) Ravn & & 1 & & 1 & & \\
\hline & & Missis. & Velamisporites magnus (Hughes y Playford) Playford & & 1 & & & & \\
\hline & 6,8 & $\begin{array}{l}\text { Dev t- } \\
\text { Missis. }\end{array}$ & Verrucosisporites depressus Winslow & & 1 & & & & \\
\hline \multirow[t]{2}{*}{$6, \mathrm{G}$} & 1,2 & Dev m-t & Verrucosisporites premnus Richardson & & 1 & & & & \\
\hline & & & Esporas no determinables & & 20 & & & 3 & \\
\hline \multicolumn{10}{|c|}{ PRASINOFITAS } \\
\hline & 1,2 & & Cymatiosphaera spp. & & 2 & & & & \\
\hline \multirow[t]{3}{*}{$6, \mathrm{~N}$} & $1,2,4,6$ & Dev & $\begin{array}{l}\text { Dictyotidium munificum (Wicander y Wood) Amenábar, di Pasquo, } \\
\text { Carrizo y Azcuy }\end{array}$ & & 1 & & & & \\
\hline & 1,2 & & Dictyotidium sp. & & 1 & & & & \\
\hline & 1,2 & & Leiosphaeridia spp. & & 5 & 4 & 1 & & \\
\hline $6, \mathrm{~K}$ & 1,6 & Dev m-t & Maranhites brasiliensis Brito emend. Burjack y Oliveira & & 4 & 2 & 1 & & \\
\hline \multirow[t]{2}{*}{$6, \mathrm{I}$} & $1,2,8$ & Dev t & Maranhites insulatus Burjack y Oliveira & 2 & 3 & 1 & & 1 & \\
\hline & 1,2 & Dev m-t & Maranhites spp. & & & & 4 & & \\
\hline \multirow[t]{4}{*}{$6, \mathrm{M}$} & 1,2 & Fra & Petrovina connata Oliveira y Burjack & & 1 & & & & \\
\hline & $1,4,6$ & Dev & Polyedryxium pharaonis Deunff ex Deunff & & 1 & & & & \\
\hline & 1,2 & & Pterospermella spp. & & 3 & & & & \\
\hline & & & ALGAS & & & & & & \\
\hline $4, \mathrm{~K}, \mathrm{P}-\mathrm{R}, \mathrm{V}$ & $1,2,4,5,8$ & S-P & Quadrisporites granulatus (Cramer) Ströther & & 8 & & & & \\
\hline \multirow[t]{2}{*}{$4, \mathrm{O}$} & $1,2,4,5$ & S-D & Quadrisporites variabilis (Cramer) Ottone y Rossello & & 3 & & & & \\
\hline & & & ACRITARCAS & & & & & & \\
\hline \multirow[t]{2}{*}{6,0} & 1,2 & Giv-Fra & Amonidium garrasinoii Ottone & & 1 & & & & \\
\hline & $1,2,4$ & Dev t & Arkonites bilixus Legault & & 1 & & & & \\
\hline \multirow[t]{2}{*}{$6, \mathrm{~F}$} & 1,2 & & Buedingisphaeridium $\mathrm{sp}$ & & & 1 & & & \\
\hline & $1,2,4$ & Dev & $\begin{array}{l}\text { Dorsenidium (D.) polyaster var. hexaster (Staplin) Stancliffe y } \\
\text { Sarjeant }\end{array}$ & & 2 & & & & \\
\hline \multirow[t]{2}{*}{$6, \tilde{N}, \mathrm{Q}$} & 1,2 & & Gorgonisphaeridium spp. & & 5 & & & & \\
\hline & $1,2,4,5$ & Dev & Navifusa bacilla (Deunff) Playford & & 1 & & & & \\
\hline $6, \mathrm{H}$ & 1,4 & Fra & Origmahapsis pachyderma Colbath & & 1 & & & & \\
\hline $6, \mathrm{~J}$ & 10 & $\mathrm{O}$ & Striatotheca frequens Burmann & & 1 & & & & \\
\hline \multirow[t]{3}{*}{$6, \mathrm{P}$} & $1,2,5,8$ & $\mathrm{~S}-\mathrm{Tc}$ & Verhyachium trispininflatum Cramer & & 1 & & & & \\
\hline & $1,2,6,8$ & $\mathrm{~S}-\mathrm{Tc}$ & Verhyachium trispinosum (Eisenack) Deunff & & 1 & & & & \\
\hline & & & Acritarcas no determinados & & & & 1 & & \\
\hline \multirow[t]{2}{*}{$6, \mathrm{R}$} & & & Quitinozoario & & 1 & & & & \\
\hline & & \multicolumn{2}{|c|}{ Total palinomorfos autóctonos y retrabajados } & 49 & 265 & 25 & 47 & 27 & 5 \\
\hline
\end{tabular}

Fig. texto 4. B. Especies retrabajadas ordenadas alfabéticamente según grupos mayores. En la tercera columna se incluye su distribución estratigráfica más consensuada, según las citas que figuran en la segunda columna. La ubicación de los palinomorfos ilustrados se consigna en la primera columna. Referencias seleccionadas: 1- di Pasquo (2003); 2- del Papa y di Pasquo (2007); 3- di Pasquo et al. (2003a, b); 4- Amenábar et al. (2006); 5- di Pasquo (2007c); 6- di Pasquo (2007b); 7- Amenábar et al. (2007); 8- Azcuy y di Pasquo (2005, 2006); 9- Lindström (1995); 10- Servais (1997). Abreviaturas: O: Ordovícico; S-D: Silúrico-Devónico; Dev m-t: Devónico Medio a Tardío; Dev t-C: Devónico Tardío-Carbonífero; Giv-Fra: GivetianoFrasniano; Missis.: Mississippiano; S-P: Silúrico-Pérmico; S-Tc.: Silúrico-Terciario. 
Fig. texto 5. Palinofacies BAFC-Pl 1492. Microfotografía en la que se observan palinomorfos autóctonos (a: grano de polen monosacado) y retrabajados (b: Retispora lepidophyta; $\mathbf{c}$ : Maranhites insulatus, ambos ilustrados en las láminas $5 \mathrm{Ay}$ 6I, respectivamente). Escala gráfica: $13 \mu \mathrm{m}$.

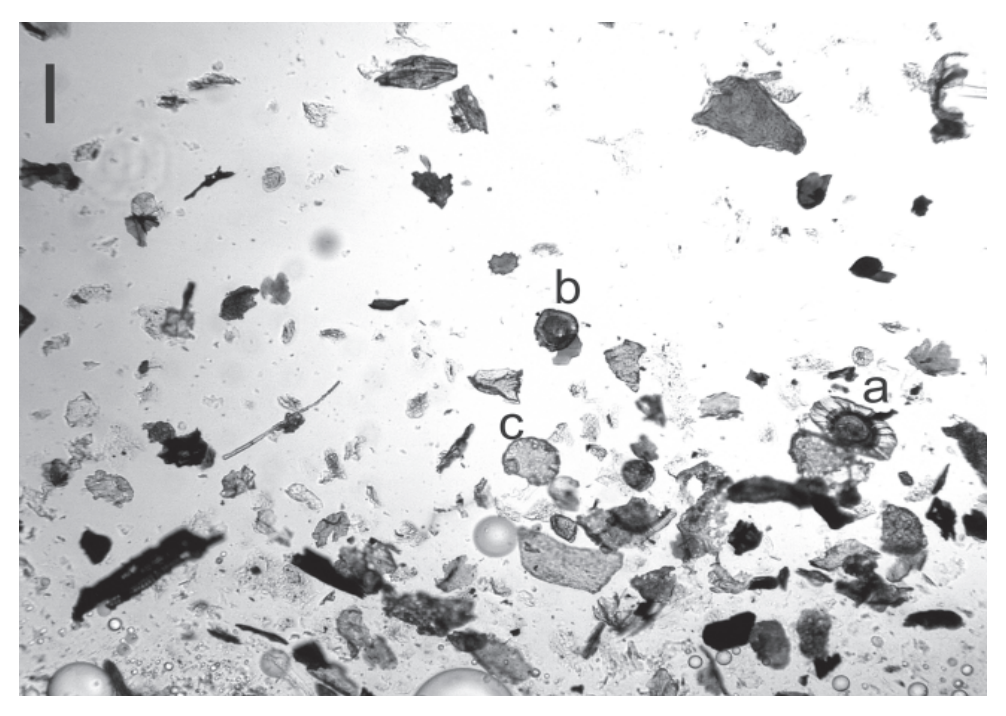

color castaño más claro a amarillo (TAI-2 a 2) (Fig. texto 5), según la escala de alteración térmica (thermal alteration index, TAI) de Utting et al. (1989). Además, se encuentran palinomorfos enteros y fragmentados tanto entre las especies autóctonas como retrabajadas; sin embargo, las primeras no presentan evidencias de piritización de la exina como sí puede observarse en la de algunos palinomorfos retrabajados con forma de pirita euhedral in situ o sus marcas (Lám. 6Q y R).

Entre los fitoclastos se destaca la presencia de cutículas formadas por células poligonales de bordes anticlinales y periclinales bien cutinizados (Lám. 4W), y otras con poros o agujeros entre sus células (Lám. 4Ñ y T), muy similares a las cutículas de semillas platispérmicas asignadas a Samaropsis newberryi (Andrews) Seward por Šimůnek y Libertín (2006, pl. 5, figs. 4-10) procedentes del Pennsylvaniano de la República Checa. Las traqueidas presentan variadas morfologías, las más frecuentes son bi a tretraseriadas con punctuaciones areoladas (Lám. 5J-N), otras menos comunes son de tipo escalariforme-espiralado (Lám. 5Ñ) o indefinidas (Lám. 5O). Si bien algunas de estas morfologías pueden ser atribuidas a diversos grupos de plantas vasculares, en el Pennsylvaniano son más comunes entre las gimnospermas en general (e.g., Stewart y Rothwell, 1993). En particular, las formas seriadas que se mencionan son similares, por ejemplo, a las traqueidas pertenecientes a troncos de Cordaitales ilustradas por Brea y Césari (1995) para el Pennsylvaniano de Argentina y por Falcon-Lang y Bashforth (2005) del Pennsylvaniano de Estados Unidos (véase también Stewart y Rothwell, 1993: 399 y $415-421)$.

\section{Discusión y Conclusiones}

\subsection{Edad de las asociaciones}

Las especies florísticas identificadas son reconocidas en las biozonas Nothorhacopteris-Botrychiopsis-Ginkgophyllum (NBG) Archangelsky et al. (1987) e Intervalo Archangelsky y Cúneo (1991), del Pennsylvaniano en las cuencas San Rafael, Paganzo y Uspallata-Iglesia (Fig. texto 3; véase además Gutiérrez, 1995; Archangelsky et al., 1996; Archangelsky, 1999; Azcuy et al., 2007). La distribución estratigráfica de las dos especies de semillas platispérmicas identificadas (Samaropsis nunezii, Cordaicarpus cesariae), se restringe al Pennsylvaniano (véase la figura texto 3 ) y se relacionan principalmente con las Coniferales y Cordaitales (e.g., Gutiérrez et al., 1992; Archangelsky, 1999, 2000; Šimůnek y Libertín, 2006). También, Ginkgophyllum diazii se restringe al Pennsylvaniano, mientras que Cordaites riojanus y Paracalamites australis se encuentran bien representadas en las asociaciones del Pennsylvaniano y se extienden al menos al Pérmico Temprano en Argentina (véase Archangelsky et al., 1996; Azcuy et al., 2007). Solamente Paracalamites australis es conocida en el Pennsylvaniano de la cuenca Paraná en Brasil (e.g., Zampirolli y Bernardes de Oliveira, 2000). 
Por otro lado, la asociación palinológica aquí estudiada se correlaciona con la Biozona $B C$ sobre la base de la presencia de especies de distribución estratigráfica restringida a dicha biozona (e.g., Dictyotriletes bireticulatus, Crucisaccites latisulcatus, Verrucosisporites morulatus, véanse las figuras texto 3 y 4A). La misma fue atribuida por di Pasquo (2003) al Bashkiriano tardío-Moscoviano y correlacionada con las Subzonas A-B de la Zona de Asociación Raistrickia densa-Convolutispora muriornata (DM) Césari y Gutiérrez (2001) y la Zona de Asociación Potonieisporites-Lundbladispora inferior (PLi) Archangelsky y Vergel (1996) en Argentina y la Zona de Intervalo Ahrensisporites cristatus (AcZ) Souza (2006) en Brasil, todas ellas referidas principalmente al Bashkiriano-Moscoviano. Se destaca, además, la incorporación de Reticulatisporites sp. cf. R. riverosii, Grumosisporites delpapae sp. nov. y Pteruchipollenites gracilis como especies autóctonas restringidas a esta biozona. Por lo tanto, la edad de la Formación Tarija en esta localidad es acotada por la asociación palinológica al Bashkiriano tardíoMoscoviano. Por ello, el hallazgo de la megaflora constituye el primer registro de la Biozona $N B G$ en la cuenca Tarija en el norte de Argentina.

\subsection{Retrabajo de palinomorfos}

El retrabajo de palinomorfos en los Grupos Macharetí y Mandiyutí fue reconocido en diversas localidades del norte de Argentina y sur de Bolivia y su significado cronológico fue tratado principalmente por di Pasquo y Azcuy (1997) y di Pasquo (2003, 2007b). La separación de especies autóctonas y retrabajadas se basó principalmente en su determinación a nivel específico y la distribución estratigráfica conocida, aunque también se tuvieron en cuenta la preservación y en menor medida la cantidad de ejemplares. Por ello, la mayoría de las especies de fitoplancton son consideradas retrabajadas. En cambio, algunos especímenes de Quadrisporites granulatus y Verhyachium trispinosum podrían ser interpretados como autóctonos, en especial aquéllos bien preservados (completos, de color castaño claro y no piritizados como se mencionó anteriormente) teniendo en cuenta que sus distribuciones estratigráficas se extienden al Pérmico y Cenozoico en América del Sur, respectivamente (véase la figura texto 4B). Asimismo, se han registrado entre las especies retrabajadas, varias especies comunes con las asociaciones del Devónico Medio y del
Carbonífero Temprano presentes en Balapuca (di Pasquo (2007b, c) como Cristatisporites matheusii, Microreticulatisporites parvirugosus y Geminospora spongiata (Fig. texto 4.B). La identificación de palinomorfos retrabajados en las asociaciones del Pennsylvaniano del norte de Argentina y sur de Bolivia estudiadas por di Pasquo (2003), aportó información que permite reconstruir de manera parcial la compleja historia geológica acaecida en el lapso entre la Formación Los Monos (Devónico Medio-Frasniano) dispuesta en general en contacto discordante con el Grupo Macharetí (Pennsylvaniano) (véase Starck y del Papa, 2006). Como ejemplo se menciona la presencia de Retispora lepidophyta en la asociación aquí estudiada, espora diagnóstica del Fameniano tardío en el mundo (Fig. texto 4B). Si bien rocas de esta antigüedad no fueron halladas en Argentina, esta especie indica que ocurrió la depositación de sedimentitas de esta edad, las cuales fueron erosionadas durante el Pennsylvaniano tal como fue expresado por di Pasquo (2003).

\subsection{Correlación}

La comparación de los datos palinológicos presentados por di Pasquo (2003) con los resultados palinológicos de del Papa y di Pasquo (2007) y los obtenidos en la localidad aquí estudiada, permiten ampliar el esquema de correlación del Grupo Macharetí propuesto por di Pasquo 2003 (Fig. texto 6). Se destaca entre las formas planctónicas autóctonas la presencia de Cymatiosphaera gondwanensis en el nivel BAFC-Pl 1480, reconocida por di Pasquo (2003) exclusivamente en el nivel BAFC-P1 1163 del tope de la Formación Tarija en el Arroyo Tuyunti. Por ello se propone una probable correlación entre ambos niveles. De igual forma, la presencia restringida de Crucisaccites latisulcatus (BAFC-Pl 1492) y Verrucosisporites morulatus (BAFC-Pl 1492 y 1494) permitió proponer la correlación de la sección aquí estudiada con otras localidades de acuerdo con la información provista por di Pasquo (2003) y del Papa y di Pasquo (2007).

\subsection{Paleoecología, tafonomía e inferencias paleoambientales}

La megaflora estudiada se compone de licofitas herbáceas, esfenofitas, cordaitales, coniferales y ginkgofitas. En concordancia, las afinidades botánicas (e.g., Balme, 1995) del conjunto de palinomor- 
O
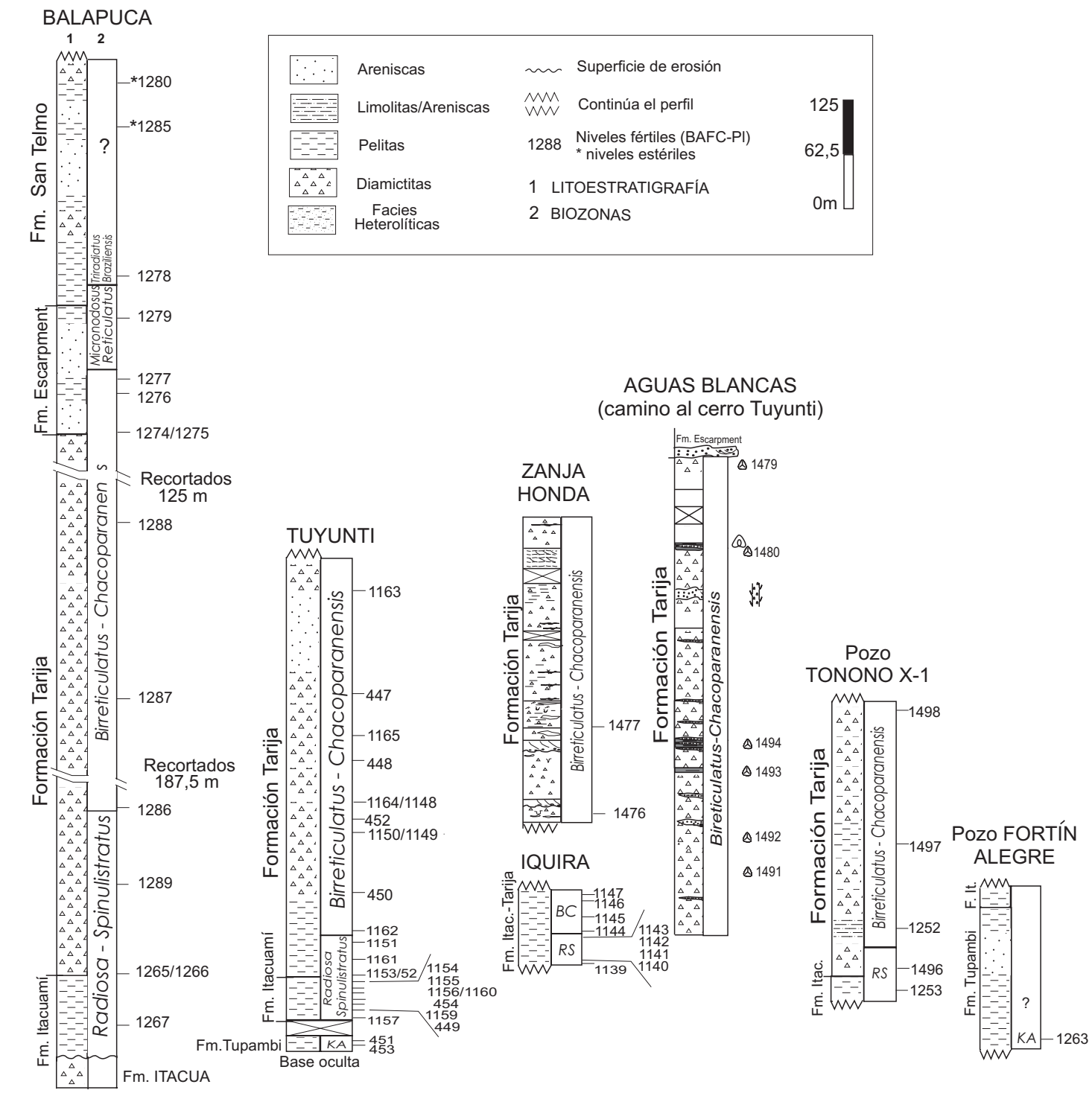

Fig. texto 6. Esquema de correlación del Grupo Macharetí teniendo como base el esquema propuesto por di Pasquo (2003), la información provista por del Papa y di Pasquo (2007), di Pasquo (2007 a, b) y los datos aquí presentados.

fos autóctonos apoyan esta variada composición florística y la amplían a grupos como las pteridofitas y pteridospermafitas (Fig. texto 4.A) no registrados entre los restos de megaflora. Por su parte, las algas clorofíceas están dominadas por Botryococcus braunii y son menos frecuentes las especies de Brazilea, Quadrisporites y Tetraporina (Fig. texto 3 y 4A). Además, varias cutículas y traqueidas dispersas podrían haber derivado principalmente de cordaitales y coniferales. De acuerdo con di Pasquo
(2003), el conjunto de palinomorfos retrabajados principalmente del Devónico aquí hallado apoya la interpretación de Starck y del Papa (2006) entre otros, que señalan que en el borde de la cuenca Tarija (norte de Argentina y sur de Bolivia) se habría desarrollado una paleogeografía montañosa conformada por rocas del Paleozoico Inferior, en la cual paleovalles habrían sido excavados en capas principalmente devónicas, y rellenados por las sedimentitas del Grupo Macharetí durante el Carbonífero y 
en particular en el Pennsylvaniano. En este contexto y considerando los requerimientos paleoecológicos de los grupos paleoflorísticos mencionados, se habría desarrollado una vegetación mesófila en áreas de montaña compuesta principalmente por Cordaitales, Coniferales primitivas y Ginkgophytas. Las esporas cingulizonadas de los géneros Cristatisporites, Kraeuselisporites y Lundbladispora, indicarían el desarrollo de una vegetación herbácea e higrófila de licofitas alrededor de lagos, lagunas y pantanos, donde también las Cordaitales podrían haber participado (e.g., ríos entrelazados y meandriformes según Falcon-Lang y Bashforth, 2005). Esporas relacionadas con las Pteridofitas (e.g., Verrucosisporites, Reticulatisporites, Dictyotriletes, Punctatisporites) suponen ambientes de sotobosque o laderas preferentemente en sombra donde la humedad es mayor. Las esfenofitas siempre representadas por el género Calamospora, se relacionan con stocks desarrollados en los márgenes de ríos y lagos. Por otro lado, las algas clorofíceas apoyan el desarrollo de cuerpos de agua dulce (e.g., Batten, 1996; Batten y Grenfell, 1996) en los cuales probablemente se depositaron las asociaciones florísticas. Cabe destacar que las rocas diamictíticas dominantes en la sección estudiada fueron interpretadas por del Papa y Martínez (2001) para esta misma sección como depósitos de flujos de detritos que habrían sido depositados en parte en cuerpos de agua $y$ en facies de canales distributarios con predominio de procesos de remoción en masa, amalgamación y migración de canales. En parte estas diamictitas habrían correspondido a facies de tilitas retrabajadas por la presencia de clastos estriados y facetados y las diamictitas estratificadas indicarían períodos de menor descarga del glaciar y retrabajo por corrientes tractivas según dichos autores. Las características sedimentológicas del depósito de areniscas de grano fino donde tallos de licofitas se disponen de manera desordenada sugieren transporte por ríos de moderada a baja energía y enterrados entre los depósitos de barra en el interior del canal. Facies subordinadas o localizadas asociadas a éstas reflejan procesos de decantación con balsaje de clastos en muchos casos semejante al depósito donde se encontraron las semillas platispérmicas (Fig. texto $2 \mathrm{~A}$ y C). Cabe destacar que el nivel BAFC-Pl 1494, donde se registran numerosas colonias de Botryococcus (Figs. texto 2A y 4A), fue obtenido de pelitas de una sección heterolítica con trazas fósiles recolectadas y estudiadas por Buatois y del Papa (2003), ubicada entre potentes bancos de diamictitas. Basado en las características iconológicas y la presencia de $B o$ tryococcus, dichos autores interpretaron la sección como un depocentro lacustre de agua dulce o bien sistemas de fiordos con gran descarga de agua dulce. Ciclos de congelamiento y descongelamiento bajo un clima húmedo como describieron también Starck y del Papa (2006), habrían sido posiblemente una de las principales causas disparadoras de procesos de retrabajo de tilitas y palinomorfos derivados de la erosión de montañas compuestas por rocas en su mayoría del Devónico (o rocas más antiguas o algo más jóvenes del Mississippiano). Estos procesos habrían sido frecuentes durante el período de depositación de la Formación Tarija debido a altas tasas de sedimentación, como fue indicado por diversos autores (e.g., López Gamundí, 1986; Starck et al., 1993; del Papa y Martínez, 2001; Buatois y del Papa, 2003; Starck y del Papa, 2006; del Papa y di Pasquo, 2007). Pese a que es frecuente que los palinomorfos de origen continental sean transportados hacia el mar, las evidencias palinológicas y paleobotánicas aquí presentadas y las características sedimentológicas analizadas en detalle por del Papa y Martínez (2001) para la Formación Tarija en esta localidad apoyan el desarrollo de un ambiente muy probablemente continental con lagos y ríos alimentados por glaciares. Según del Papa y di Pasquo (2007), la Formación Tarija estaría comprendida en el ciclo II glacial-periglacial (equivalente a las facies II y III de del Papa y Martínez, 2001), mientras que las subyacentes formaciones Tupambi e Itacuamí se habrían depositado en un ciclo I no glacial.

\section{Agradecimientos}

Un especial agradecimiento a los Dres. C. Azcuy (CONICET), C. del Papa (UNAS) y el Licenciado D. Starck (Tecpetrol S.A.) por ceder los materiales aquí estudiados, a la Licenciada B. Cariglino por haber colaborado con la autora en la medición de las licofitas, al Licenciado G. Holfeltz por el procesamiento de las muestras palinológicos y en particular a los revisores de la presente contribución, Doctores P. Gutiérrez, E. Pérez D’Angelo y uno anónimo. Este trabajo fue financiado por la Agencia Nacional de Promoción Científica y Tecnológica PICTR 00313/03, el Consejo Nacional de Investigaciones Científicas y Técnicas PIP 5518 CONICET, la Universidad de Buenos Aires UBACYT X 136 y el Ministerio de Educación y Ciencia de España CGL2006-07376/BTE. 


\section{Referencias}

Amenábar, C.R.; di Pasquo, M.; Carrizo, H.; Azcuy, C.L. 2006. Palynology of the Chigua (Devonian) and Malimán (Carboniferous) formations in Del Volcán Range, San Juan Province, Argentina. Part I. Paleomicroplankton and acavate smooth and ornamented spores. Ameghiniana 43 (2): 339-375.

Amenábar, C.R.; di Pasquo, M.; Carrizo, H.; Azcuy, C.L. 2007. Palynology of the Chigua (Devonian) and Malimán (Carboniferous) formations in Del Volcán Range, San Juan Province, Argentina. Part II. Cavate, pseudosaccate and cingulizonate spores. Ameghiniana 43 (3): 547-564.

Archangelsky, A. 1999. Semillas del Paleozoico Superior de Argentina: su utilización bioestratigráfica. Ameghiniana 36 (4): 465-475.

Archangelsky, A. 2000. Estudio sobre semillas neopaleozoicas de Argentina. Boletín de la Academia Nacional de Ciencias 64: 79-115.

Archangelsky, S.; Arrondo, O.G. 1974. Paleophytologia Kurtxiana. III. 11. Dos especies de Ginkgophyllum del Paleozoico Superior de la Provincia de La Rioja. Ameghiniana 11 (4): 357-365.

Archangelsky, S.; Cúneo, N.R. 1991. The neopaleozoic succession from northwestern Argentina. A new perspective. In Gondwana Seven Proceedings (Ulbrich, H.; Rocha-Campos, A.C.; editors). Instituto de Geoçiências, Universidade de Sâo Paulo: 469-481. Sâo Paulo.

Archangelsky, S.; Leguizamón, R.R. 1980. El registro de Ginkgophyllum diazii en el Carbónico de Sierra de los Llanos. Provincia de La Rioja. Boletín de la Academia Nacional de Ciencias 53 (3-4): 211-219.

Archangelsky, S.; Vergel, M.M. 1996. Cuenca Chacoparanense. Paleontología, bioestratigrafía y paleocología. In El Sistema Pérmico en la República Argentina y en la República Oriental del Uruguay (S. Archangelsky; editor). Academia Nacional de Ciencias: 40-44. Córdoba.

Archangelsky, S.; Azcuy, C.L.; Césari, S.N.; González, C.R.; Sabattini, N. 1987. Correlación general de biozonas. In El Sistema Carbonífero en la República Argentina (Archangelsky, S.; editor). Academia Nacional de Ciencias: 281-292. Córdoba.

Archangelsky, S.; Azcuy, C.L.; Wagner, R. 1981. Three dwarf lycophytes from the Carboniferous of Argentina. Scripta Geologica 64: 1-35.

Archangelsky, S.; González, C.R.; Cúneo, R.; Sabattini, N.; Césari, S.N.; Aceñolaza, F.G.; García, G.B.; Buatois, L.; Ottone, E.; Mazzoni, A.F.; Hünicken, M.A.; Gutiérrez, P.R. 1996. Paleontología, bioestratigrafía y paleoecología de las cuencas Paganzo, Calingasta-Uspallata, Río Blanco y San Rafael. In El Sistema Pérmico en la República Argentina y en la República Oriental del Uruguay (Archangelsky, S.; editor). Academia Nacional de Ciencias: 177-201. Córdoba.

Azcuy, C.L.; di Pasquo, M.M. 2005. Early Carboniferous palynoflora of the Ambo Formation, Pongo de Mainique, Perú. Review of Palaeobotany and Palynology 134 (3-4): 153-184.

Azcuy, C.L.; di Pasquo, M.M. 2006. Additional systematic information on the Early Carboniferous palynomorphs from the Ambo Formation, Pongo de Mainique, Perú. Revista Brasileira de Paleontología 9 (1): 41-52.

Azcuy, C.L.; Beri,A.; Bernardes-de-Oliveira, M.E.C.; Carrizo, H.A.; di Pasquo, M.; Díaz Saravia, P.; González, C.; Iannuzzi, R.; Lemos, V.B.; Melo, J.H.G.; Pagani, A.; Rohn, R.; Rodríguez Amenábar, C.; Sabattini, N.; Souza, P.A.; Taboada, A.; Vergel, M.M. 2007. Bioestratigrafía del Paleozoico Superior de América del Sur: primera etapa de trabajo hacia una nueva propuesta cronoestratigráfica. Asociación Geológica Argentina, Serie D, Publicación Especial 11: 9-65.

Balme, B.E. 1995. Fossil in situ spores and pollen grains: an annotated catalogue. Review of Palaeobotany and Palynology 87: 81-323.

Batten, D.J. 1996. 26. Palynofacies. In Palynology: principles and applications (Jansonius, J.; McGregor, D.C.; editors). American Association of Stratigraphic Palynologists Foundation 3: 1011-1084.

Batten, D.J.; Grenfell, H.R. 1996. 7D. Botryococcus. In Palynology: principles and applications (Jansonius, J.; McGregor, D.C.; editors). American Association of Stratigraphic Palynologists Foundation 1: 205-214.

Brea, M.; Césari, S. 1995. An anatomically preserved stem from the Carboniferous of Gondwana:Phyllocladopitys petriellae Brea and Césari sp. nov. Review of Palaeobotany and Palynology 86: 315-323.

Buatois, L.; del Papa, C. 2003. Trazas fósiles de la cuenca Tarija, Pennsylvaniano del norte argentino: aspectos icnológicos de la glaciación gondwánica. In Reunión Argentina de Icnología, No. 4, Reunión de Icnología del Mercosur, No. 2 (Buatois, L.; Mángano, G.; editores). Asociación Paleontológica Argentina, Publicación Especial 9: 119-130.

Carrizo, H.A.; Azcuy, C.L. 1998. El perfil del cerro Mudadero y su flora fósil. Bolsón de Jagüé, Provincia de La Rioja. Argentina. Acta Geológica Lilloana 18 (1): 81-99.

Césari, S.N.; Gutiérrez, P.R. 2001. Palynostratigraphic study of the Upper Paleozoic central-western Argentinian sequences. Palynology 24: 113-146. Dallas.

Davydov, V.; Wardlaw, B.R.; Gradstein, F.M. 2004. The Carboniferous Period. In A Geologic Time Scale (Gradstein, F.M.; Ogg, J.G.; Smith, A.G.; editors). Cambridge University Press, chapter 15: 222-248.

del Papa, C.E.; Martínez, L. 2001. Sedimentación lacustre glaci-dominada en la Formación Tarija (Carbonífero), sierra de Aguaragüe, noroeste argentino. Revista de la Asociación Argentina de Sedimentología 8 (1): 61-76.

del Papa, C.; di Pasquo, M.M. 2007. Palaeoenvironmental interpretation and palynology of outcrop and subsurface sections of the Tarija Formation (Upper Carboniferous), Northwestern Argentina. Journal of South American Earth Sciences 23: 99-119. 
Dettmann, M. 1963. Upper microfloras from southeastern Australia. Proceedings of the Royal Society of Victoria 77: 1-148.

di Pasquo, M.M. 2003. Avances sobre palinología, bioestratigrafía y correlación de las asociaciones presentes en los Grupos Macharetí y Mandiyutí, Neopaleozoico de la Cuenca Tarija, Provincia de Salta, Argentina. Ameghiniana 40: 3-32.

di Pasquo, M.M. 2007a. Update and importance of the Carboniferous and Permian paleontological records of the Tarija Basin. In European Meeting on Paleontology and Stratigraphy of Latin American, No. 4, Instituto Geológico y Minero de España, Serie Cuadernos del Museo Geominero 8: 107-112. Madrid.

di Pasquo, M.M. 2007b. Asociaciones palinológicas presentes en las formaciones Los Monos (Devónico) e Itacua (Carbonífero Inferior) en el perfil de Balapuca, sur de Bolivia. Parte 2. Formación Itacua e interpretación estratigráfica y cronología de las formaciones Los Monos e Itacua. Revista Geológica de Chile 34 (2): 163-198.

di Pasquo, M.M. 2007c. Asociaciones palinológicas en las formaciones Los Monos (Devónico) e Itacua (Carbonífero Inferior) en Balapuca (Cuenca Tarija), sur de Bolivia. Parte 1. Geología, estratigrafía y asociaciones de la Formación Los Monos. Revista Geológica de Chile 43 (1): 98-137.

di Pasquo, M.M.; Azcuy, C.L. 1997. Palinomorfos retrabajados en el Carbonífero Tardío de la Cuenca Tarija (Argentina): su aplicación a la datación de eventos diastróficos. Revista Universidade Guarulhos, Série Geociências 2 (No. especial): 28-42.

di Pasquo, M.M.; Azcuy, C.A.; Souza, P.A. 2003a. Palinología del Carbonífero Superior del Subgrupo Itararé en Itaporanga, Cuenca Paraná, Estado de São Paulo, Brasil. Parte 1: sistemática de esporas y paleofitoplancton. Ameghiniana 40 (3): 277-296.

di Pasquo, M.M.; Azcuy, C.A.; Souza, P.A. 2003b. Palinología del Carbonífero Superior del Subgrupo Itararé en Itaporanga, Cuenca Paraná, Estado de São Paulo, Brasil. Parte 2: sistemática de polen y significado paleoambiental y estratigráfico. Ameghiniana 40 (3): 297-313.

Falcon-Lang, H.J.; Bashforth, A.R. 2005. Morphology, anatomy, and upland ecology of large cordaitalean trees from the Middle Pennsylvanian of Newfoundland. Review of Palaeobotany and Palynology 135: 223-243.

García, G.B. 1990. Cordaitales y semillas platispérmicas de la Formación El Imperial (Paleozoico Superior), cuenca San Rafael, República Argentina. Ameghiniana 27 (3-4): 239-249.

Gutiérrez, P.R. 1995. Nuevos registros paleoflorísticos para la Formación Agua Colorada, Carbonífero Superior, en el sector sudoriental de la sierra de Famatina, Provincia de La Rioja, Argentina. Ameghiniana 32 (2): 111-128.
Gutiérrez, P.R.; Ganuza, D.G.; Morel, E.; Arrondo, O.G. 1992. Los géneros Cordaicarpus Geinitz, Cornucarpus Arber y Samaropsis Geoppert (semillas platispérmicas) en el Neopaleozoico argentino. Ameghiniana 29 (1): 49-68.

Lindström, S. 1995. Early Permian palynostratigraphy of the northern Heimefrontfjella mountain-range, Dronning Maud Land, Antarctica. Review of Palaeobotany and Palynology 89: 359-415.

López Gamundí, O.R. 1986. Sedimentología de la Formación Tarija, Carbonífero de la sierra de Aguaragüe, Provincia de Salta. Revista de la Asociación Geológica Argentina 41: 334-355.

Potonié, R. 1970. Synopsis der Gattungen der Sporae dispersae. V. Teil: Nachtrage zu allen Gruppen (Turmae). Beihefte zum geologischen Jahrbuch 87: 1-222.

Rigby, J.F. 1966. The Lower Gondwana Floras of the Perth and Collie Basins, Western Australia. Palaeontographica Abt. B 118 (4-6): 123-126.

Servais, T. 1997. The Ordovician Arkonia-Striatotheca acritarch plexus. Review of Palaeobotany and Palynology 98: 47-79.

Šimůnek, Z.; Libertín, M. 2006. Cordaites schatzlarensis sp. nov. and Samaropsis newberryi (Andrews) Seward from the Westphalian (Carboniferous) of the Zacler area (Czech Republic). Review of Palaeobotany and Palynololgy 138: 43-62.

Smith, A.H.V.; Butterworth, M.A. 1967. Miospores in the coal sems of the Carboniferous of Great Britain. The Palaeontological Association, Special Paper in Palaeontology 1: 1-324.

Souza, P.A. 2006. Late Carboniferous palynostratigraphy of the Itararé Subgroup, northeastern Paraná Basin, Brazil. Review Palaeobotany Palynology 138: 9-29.

Starck, D.; del Papa, C. 2006. The northwestern Argentina Tarija Basin-Stratigraphy, depositional systems and controlling factors in a glaciated basin. Journal of South American Earth Sciences 22: 169-184.

Starck, D.; Gallardo, E.; Schulz, A. 1993. Neopaleozoic stratigraphy of the Sierras Subandinas Occidentales and Cordillera Oriental, Argentina. In International Congress of Carboniferous-Permian, No. 12, Comptes Rendus 2: 353-372. Buenos Aires (1991).

Stewart, W.; Rothwell, G. 1993. Palaeobotany and the evolution of plants. Cambridge University Press: 521. New York.

Utting, J.; Goodarzi, F.; Dougherty, B.J.; Henderson, C.M. 1989. Thermal maturity of Carboniferous and Permian rocks of the Sverdrup Basin. Canadian Arctic Archipelago. Geological Survey Canada 89 (19): 1-20.

Zampirolli, A.P.; Bernardes de Oliveira, M.E. 2000. O gênero Paracalamites Zalessky 1927, na tafoflora de Itapeva, Carbonífero Superior do Subgrupo Itararé, Grupo Tubarâo, sudoeste do Estado Sâo Paulo, Brasil. Revista do Instituto Geológico de Sâo Paulo 21 (1-2): 7-15. 
LÁMINAS 1-6 


\section{LÁMINA 1}

Megafloras registradas en la Formación Tarija, Arroyo Aguas Blancas, Sierra de Aguaragüe, Provincia de Salta, Argentina.

\section{Figuras}

A, D y E Samaropsis nunezii García emend. Archangelsky. A y D. BAFC-Pb 16358 a, molde. Escala en F: 7 mm. E. BAFC-Pb 16360. Escala: 5 mm.

B y C Cordaicarpus cesariae Gutiérrez, Ganuza, Morel y Arrondo emend. Archangelsky. B. BAFC-Pb 16357 a, molde. Escala: 5 mm. C. BAFC-Pb 16361 a, molde. Escala: 5 mm.

F. Ginkgophyllum sp. cf. G. diazii Archangelsky y Arrondo. BAFC-Pb 16355. Escala: 2 cm.

G-J Cordaites riojanus Archangelsky y Leguizamón. G. BAFC-Pb 16361 a. Escala: 5 mm. H. BAFC-Pb 16361 a. Escala: $1 \mathrm{~cm}$. I. BAFC-Pb 16355. Escala: $5 \mathrm{~mm}$. J. Detalle de la venación de la figura I. Escala: 2,5 mm.

K Paracalamites australis Rigby emend. Zampirolli y Bernardes de Oliveira. BAFC-Pb 16360. Escala: 2 $\mathrm{cm}$. 
LÁMINA 1
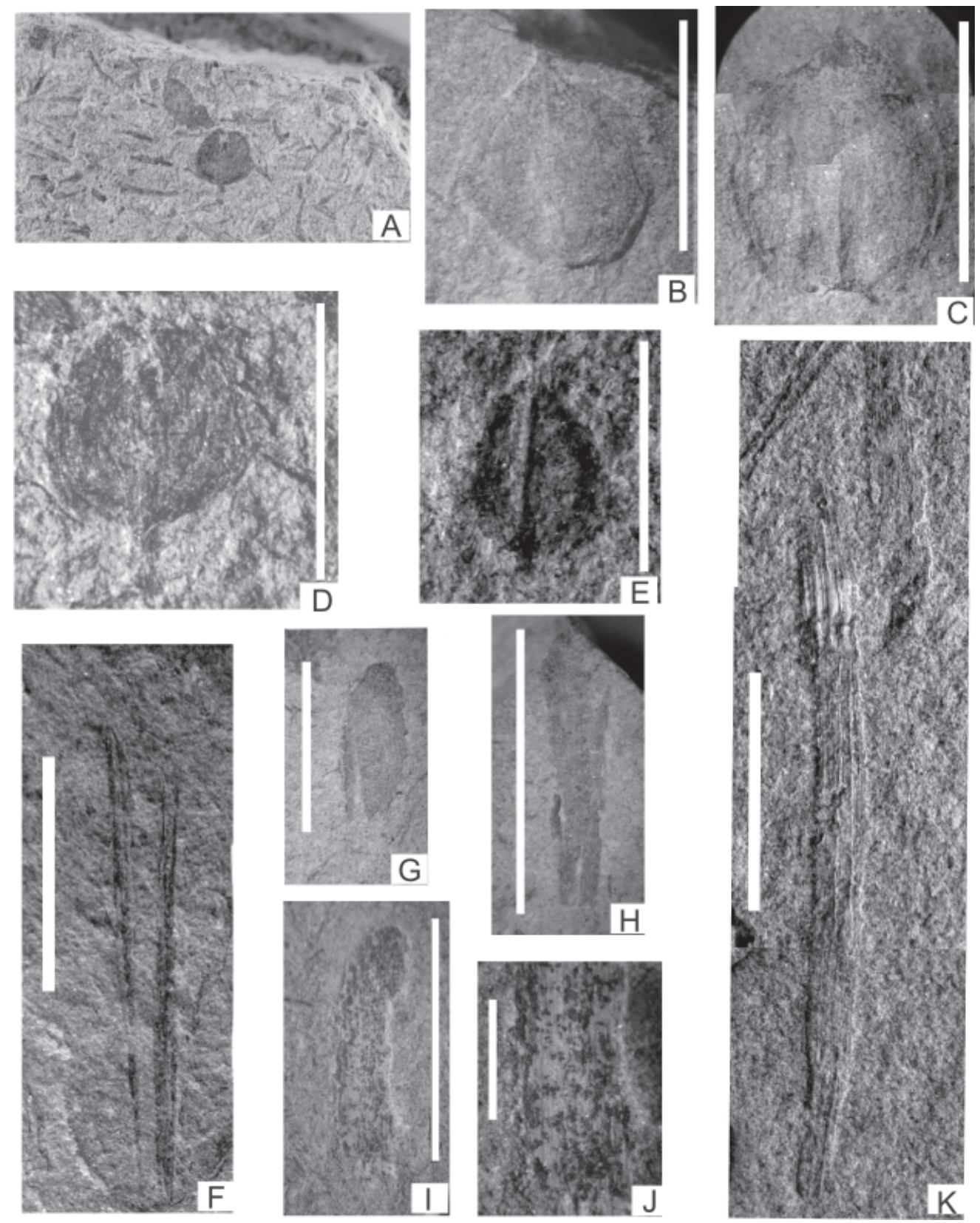


\section{LÁMINA 2 \\ Malanzania starckii sp. nov.}

Quebrada Aguas Blancas, Sierra de Aguaragüe, Provincia de Salta, Argentina p. 99.

\section{Figuras}

A y B Paratipo BAFC-Pb 16375. Cast. La figura A es un detalle de la figura B (indicado por la flecha) donde se observan bases foliares en disposición lepidodendroide. En el recuadro menor se destaca una micrófila en el margen del tallo. Escala $=1,3 \mathrm{~cm}$.

C Paratipo BAFC-Pb 16387. Cast. Tallo con micrófilas. Escala $=1 \mathrm{~cm}$.

D Paratipo BAFC-Pb 16376. Molde externo. Se destaca la base de una micrófila en el margen. Escala = 1,2 $\mathrm{cm}$.

E y G Holotipo BACF-Pb 16369. Cast. E. En el recuadro se muestra una reconstrucción esquemática de dos bases foliares con sus micrófilas ubicadas en ambos márgenes del tallo y el ángulo de filotaxis. Escala 1,5 cm. G. Detalle de la porción del tallo en el recuadro en E.

F Paratipo BAFC-Pb 16380. Compresión carbonosa parcial. Fragmento de tallo con micrófilas. Escala = 1,4 $\mathrm{cm}$.

H e I Paratipo BAFC-Pb 16368. Cast. Ejemplar ilustrado en la Fig. texto 2B. I. Detalle de H donde se observan bases foliares poco marcadas y micrófilas en el margen. Escala $=1,6 \mathrm{~cm}$. 
LÁMINA 2
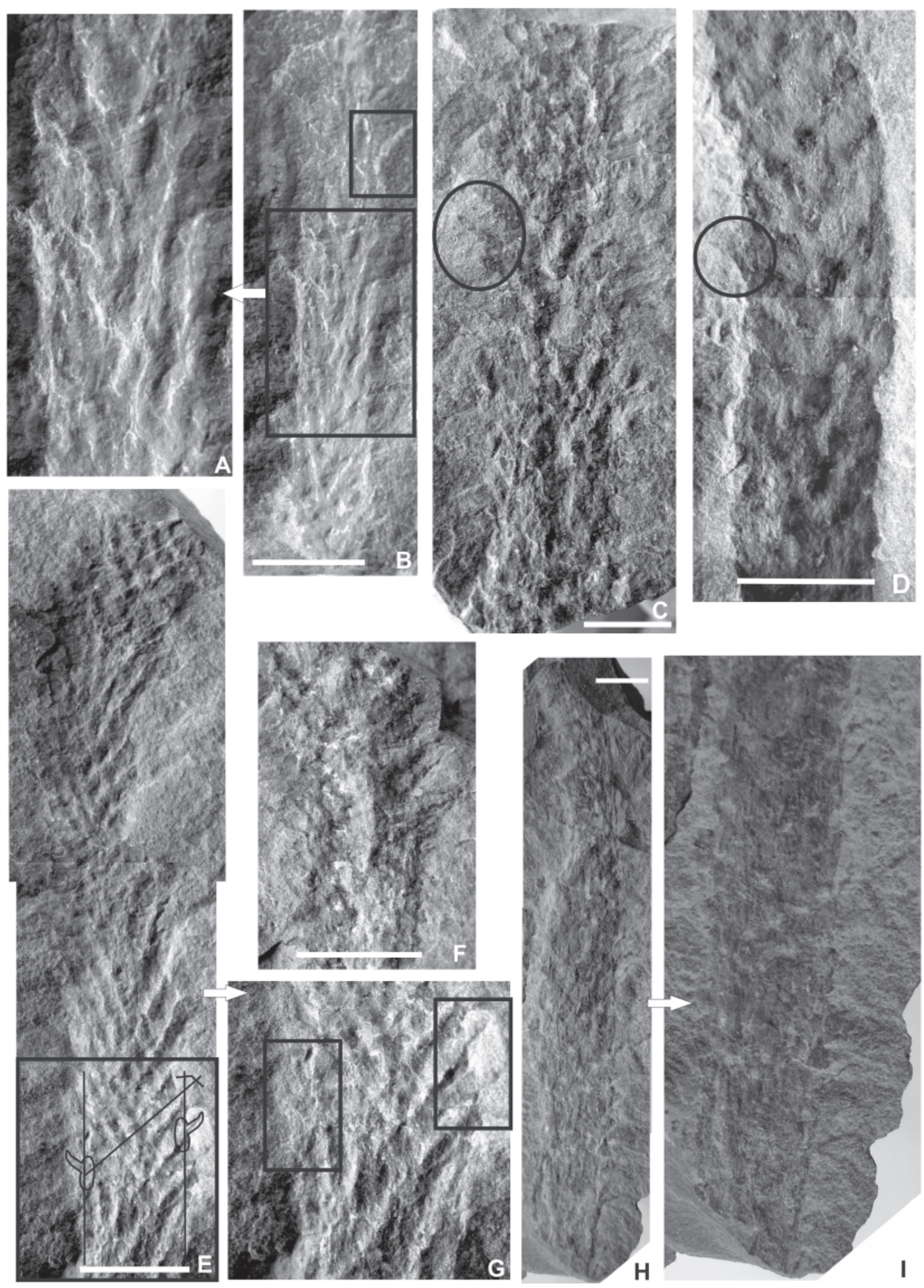


\section{LÁMINA 3}

Esporas autóctonas registradas en la Formación Tarija, arroyo Aguas Blancas, Sierra de Aguaragüe, Provincia de Salta, Argentina.

Escala gráfica: A, G-H, O $20 \mu \mathrm{m}, \mathrm{B}-\mathrm{D}, \mathrm{I}-\mathrm{J}, \mathrm{N}, \mathrm{P}-\mathrm{S} 15 \mu \mathrm{m}, \mathrm{E}-\mathrm{F}, \mathrm{K}-\mathrm{N} 10 \mu \mathrm{m}$.

\section{Figuras}

A Reticulatisporites riverosii Ottone. BAFC-P1 1479(1) J23/3.

B Dictyotriletes bireticulatus (Ibrahim) Potonié y Kremp emend. Smith y Butterworth. BAFC-Pl 1480(2) V23/3.

C Reticulatisporites polygonalis (Ibrahim) Loose. BAFC-P1 1492(1) R39.

D Verrucosisporites morulatus (Knox) Potonié y Kremp emend. Smith y Butterworth. BAFC-Pl 1492(1) $\mathrm{X} 43$.

E Cyclogranisporites minutus Bhardwaj. BAFC-P1 1492(1) S26/4.

F Velamisporites australiensis (Playford y Helby) di Pasquo, Azcuy y Souza. BAFC-P1 1491(1) N27/1.

G Endosporites rhytidosaccus Menéndez y Azcuy. BAFC-Pl 1491(2) N37/3.

$\mathrm{H} \quad$ Spelaeotriletes ybertii (Marques Toigo) Playford y Powis emend. Playford et al. BAFC-P1 1480(1) M59/2.

I Pustulatispories papillosus (Knox) Potonié y Kremp. BAFC-P1 1492(1) H51.

J Lundbladispora riobonitensis Marques Toigo y Picarelli. BAFC-P1 1480(1) W34.

K Vallatisporites ciliaris (Luber) Sullivan. BAFC-P1 1492(1) G28.

L Cristatisporites stellatus (Azcuy) Gutiérrez y Limarino. BAFC-P1 1492(1) O41.

M-N Grumosisporites delpapae sp. nov. M. Paratipo. BAFC-P1 1491(1) D27. N. Holotipo. BAFC-P1 1494(2) P37/4.

N $\quad$ Stenozonotriletes menendezii Azcuy. BAFC-Pl 1491(2) D42/3.

O Cristatisporites menendezii (Menéndez y Azcuy) Playford emend. Césari. BAFC-P1 1492(1) E36.

P Cristatisporites scabiosus Menéndez. BAFC-P1 1491(3) O44/2.

Q Verrucosisporites verrucosus (Ibrahim) Ibrahim. BAFC-P1 1480(1) B25.

R Cristatisporites spinosus (Menéndez y Azcuy) Playford 1978 emend. Césari. BAFC-P1 14890(3) O39.

S Calamospora hartungiana Schopf en Schopf, Wilson y Bentall. BAFC-P1 1492(1) L34/1. 

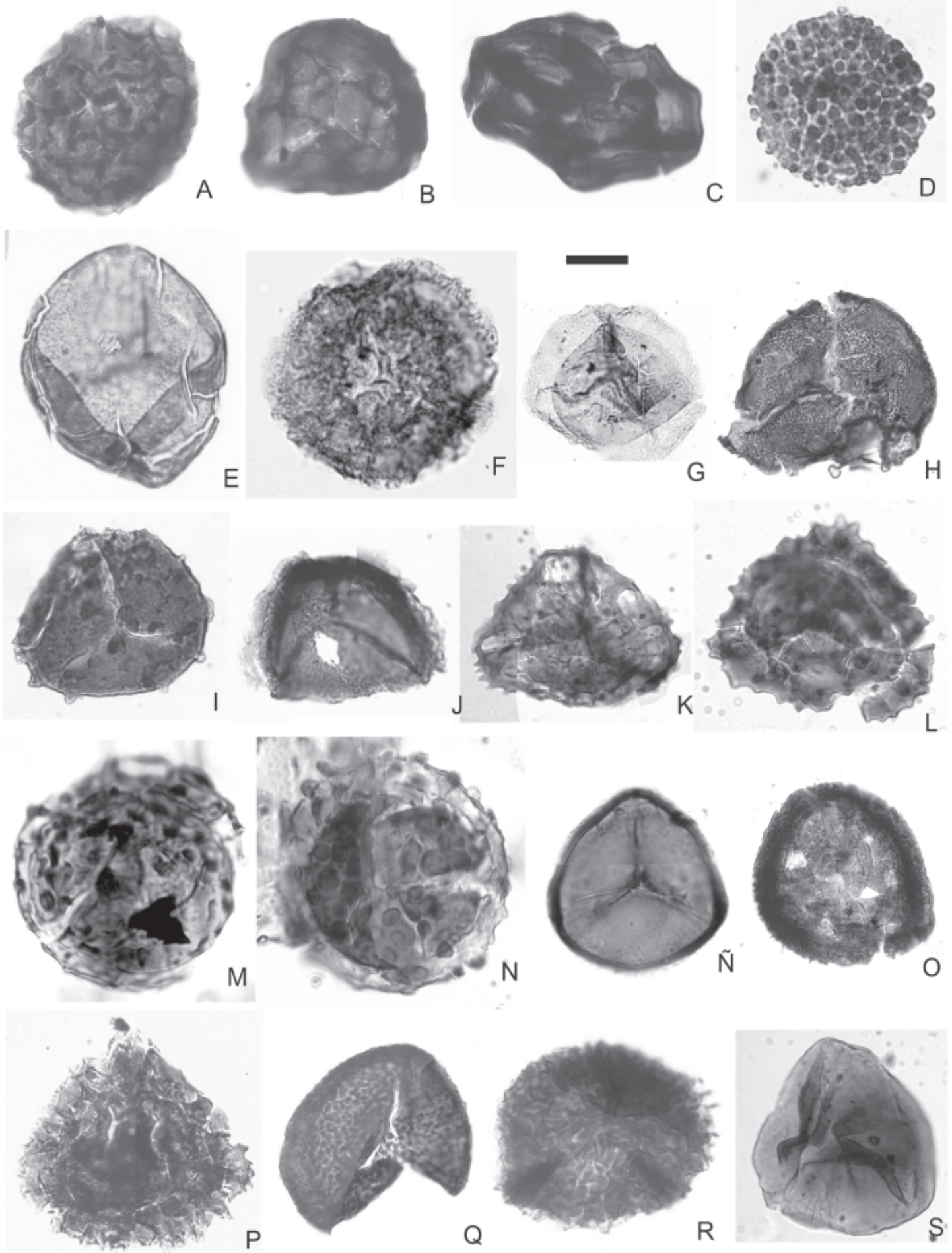


\section{LÁMINA 4}

Polen, algas y cutículas registradas en la Formación Tarija.

Escala gráfica: A, I, K-P, R, U, V $20 \mu \mathrm{m}, \mathrm{B}-\mathrm{G}, \mathrm{W} 40 \mu \mathrm{m}, \mathrm{H}, \mathrm{Q} 10 \mu \mathrm{m}, \mathrm{J}, \mathrm{S} 15 \mu \mathrm{m}, \tilde{\mathrm{N}}$ 27,5 $\mu \mathrm{m}, \mathrm{T} 17 \mu \mathrm{m}$.

\section{Figuras}

A Cannanoropollis janakii Potonié y Sah. BAFC-Pl 1492(1) K34/3.

B Crucisaccites latisulcatus Lele y Maithy. BAFC-P1 1492(1) G34/4.

C Plicatipollenites malabarensis (Potonié y Sah) Foster. BAFC-P1 1492(1) O32/2.

D Potonieisporites neglectus Potonié y Lele. BAFC-P1 1492(1) E33.

E Potonieisporites congoensis Bose y Maheshwari. BAFC-P1 1492(1) Q28.

F, J, L-N Botryococcus braunii Kützing. F. BAFC-P1 1493(1) Y53/1. J. BAFC-Pl 1491(3) G55. L. BAFC-P1 1494(1) N45/1. M. BAFC-P1 1492(1) N33/1. N. BAFC-Pl 492(1) F53/3.

G Potonieisporites novicus Bharadwaj emend. Poort y Veld. BAFC-P1 1492(1) V24/4.

H Cymatiosphaera gondwanensis (Tiwari) Backhouse. BAFC-P1 1480(2) T19/1.

I Pteruchipollenites sp. BAFC-Pl 1479(4) G41.

K, P-R, V Quadrisporites granulatus (Cramer) Ströther. K. BAFC-P1 1492(1) G49. P. BAFC-P1 1492(1) S30/2. Q. BAFC-Pl 1492(1) H34/3. R. BAFC-Pl 1492(1) L43/2. V. BAFC-Pl 1492(1) G34/1.

Ñ, T, W Cutículas. Ñ. BAFC-P1 1480(3) M25/3. T. BAFC-P1 1491(3) E34. Presencia de poros o agujeros entre células poligonales. W. BAFC-P1 1480(3) W27/1. Se observan células poligonales de bordes anticlinales y periclinales bien cutinizados.

O Quadrisporites variabilis (Cramer) Ottone y Rossello. BAFC-P1 1492(1) X38.

S Brazilea scissa (Balme y Hennelly) Foster. BAFC-P1 1492(1) Z29/2.

U Quadrisporites horridus Hennelly ex Potonié y Lele. BAFC-P1 1492(1) D41/3. 
LÁMINA 4
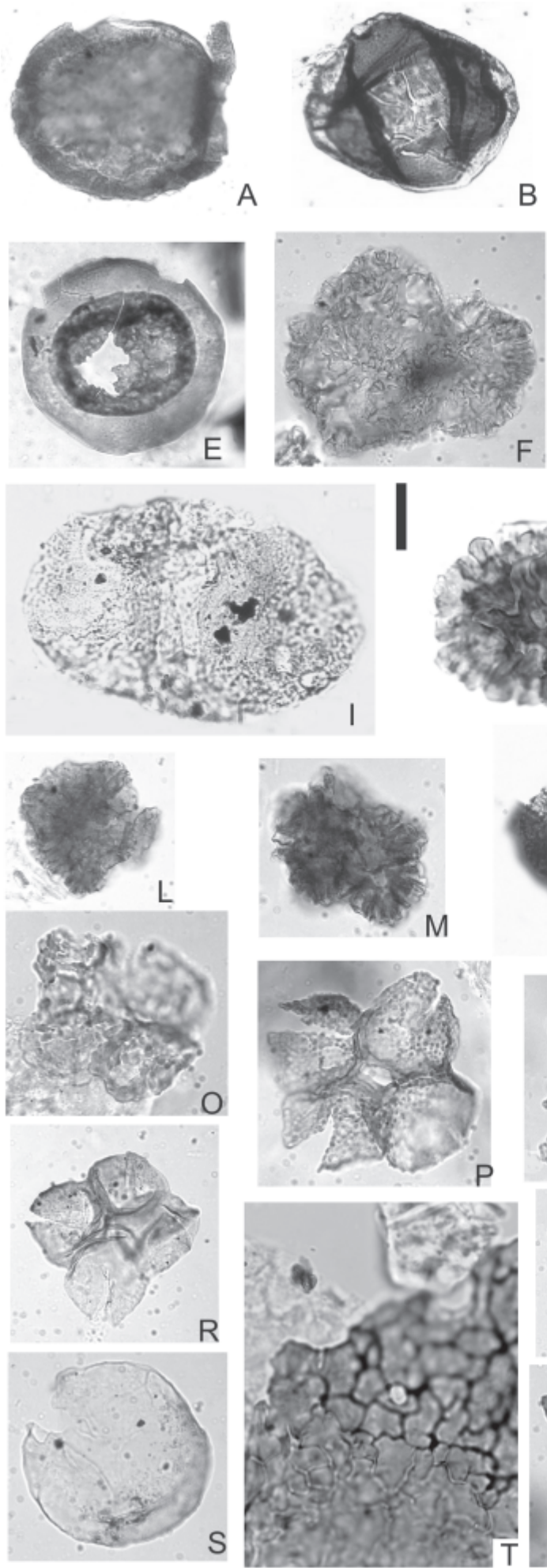
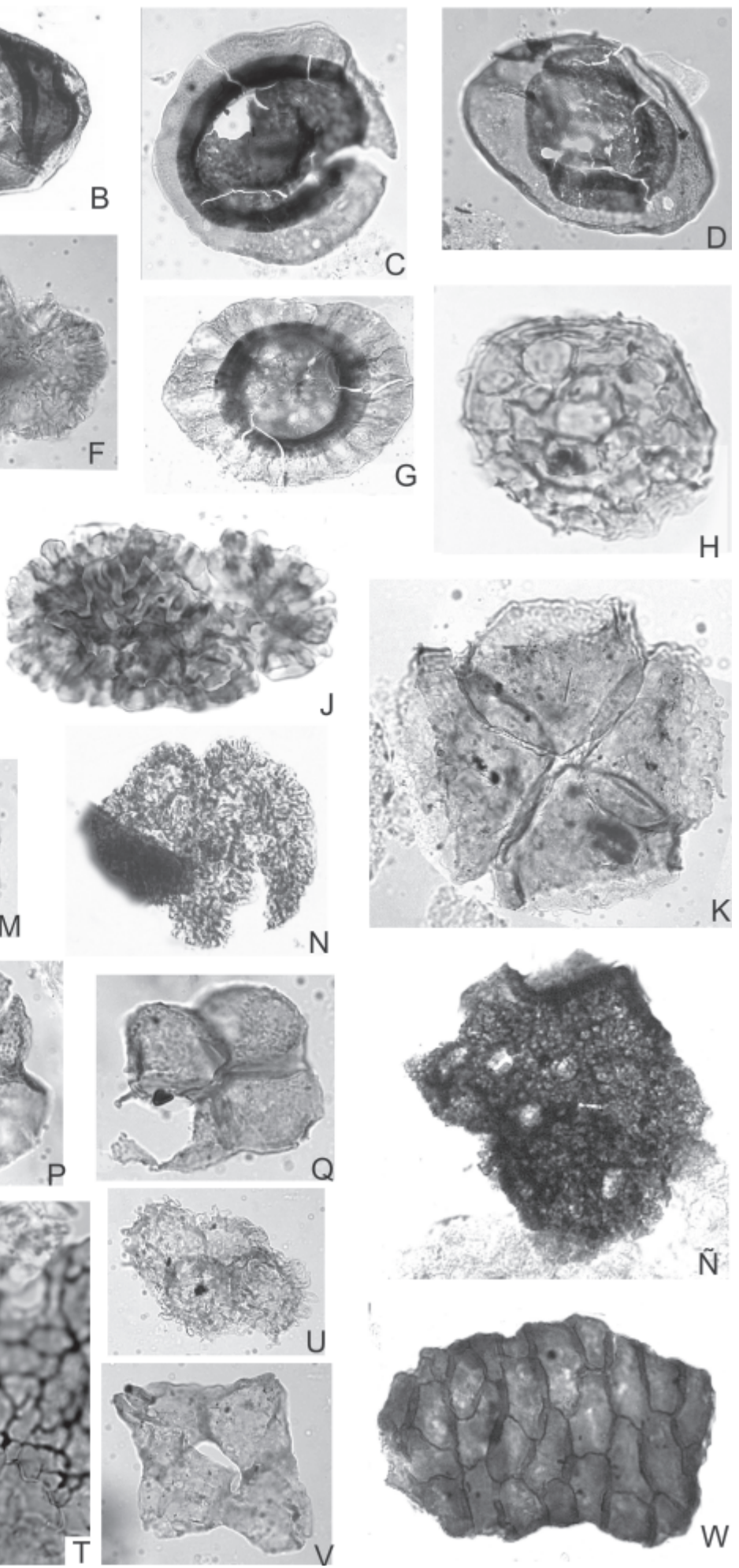


\title{
LÁMINA 5
}

Esporas retrabajadas y leños presentes en la Formación Tarija.

Escala gráfica: A, E, F, H, I $10 \mu \mathrm{m}$, B-D $15 \mu \mathrm{m}, \mathrm{G}, \mathrm{J}-\mathrm{O} 20 \mu \mathrm{m}$.

\author{
A Retispora lepidophyta (Kedo) Playford. BAFC-P1 1492(1) H28/1. \\ B Convolutispora circumvallata Clayton. BAFC-P1 1492(1) U53. \\ C Grandispora daemonii Loboziak, Streel y Burjack. BAFC-P1 1491(2) N42. \\ D Geminospora tuberculata var. tuberculata McGregor. BAFC-P1 1491(2) Q38. \\ E Cymbosporites cyathus Allen. BAFC-P1 1492(1) P43. \\ F Rugospora minuta Neves y Ioannides. BAFC-P1 1492(1) T35/3-4. \\ G Kraeuselisporites explanatus (Luber) Azcuy y di Pasquo. BAFC-P1 1494(1) S43/1. \\ H Emphanisporites orbicularis Turnau. BAFC-P1 1492(1) Z25/2. \\ I Geminospora lemurata Balme emend. Playford. BAFC-P1 1494(1) C43/3. \\ J-O Leños. J. BAFC-P1 1480(3) E53. Forma bi-triseriada con puntuaciones areoladas y poros inclinados. K. \\ BAFC-P1 1480(3) L24/3. Forma mono a biseriada con puntuaciones areoladas y poros circulares e incli- \\ nados. L. BAFC-P1 1492(1) J31/2. Forma tetraseriada con puntuaciones areoladas y poros inclinados. M. \\ BAFC-P1 1494(1) E23/2. Forma tetraseriada con puntuaciones areoladas y poros circulares e inclinados. \\ N. BAFC-P1 1492(1) R29/3. Forma mono a biseriada con puntuaciones areoladas y poros circulares e \\ inclinados. N. BAFC-P1 1480(1) R33. Traqueida con engrosamientos escalariformes-espiralados. O. \\ BAFC-P1 1492(1) H27/2. Fragmento semejante a campos de cruzamiento.
}


LÁMINA 5
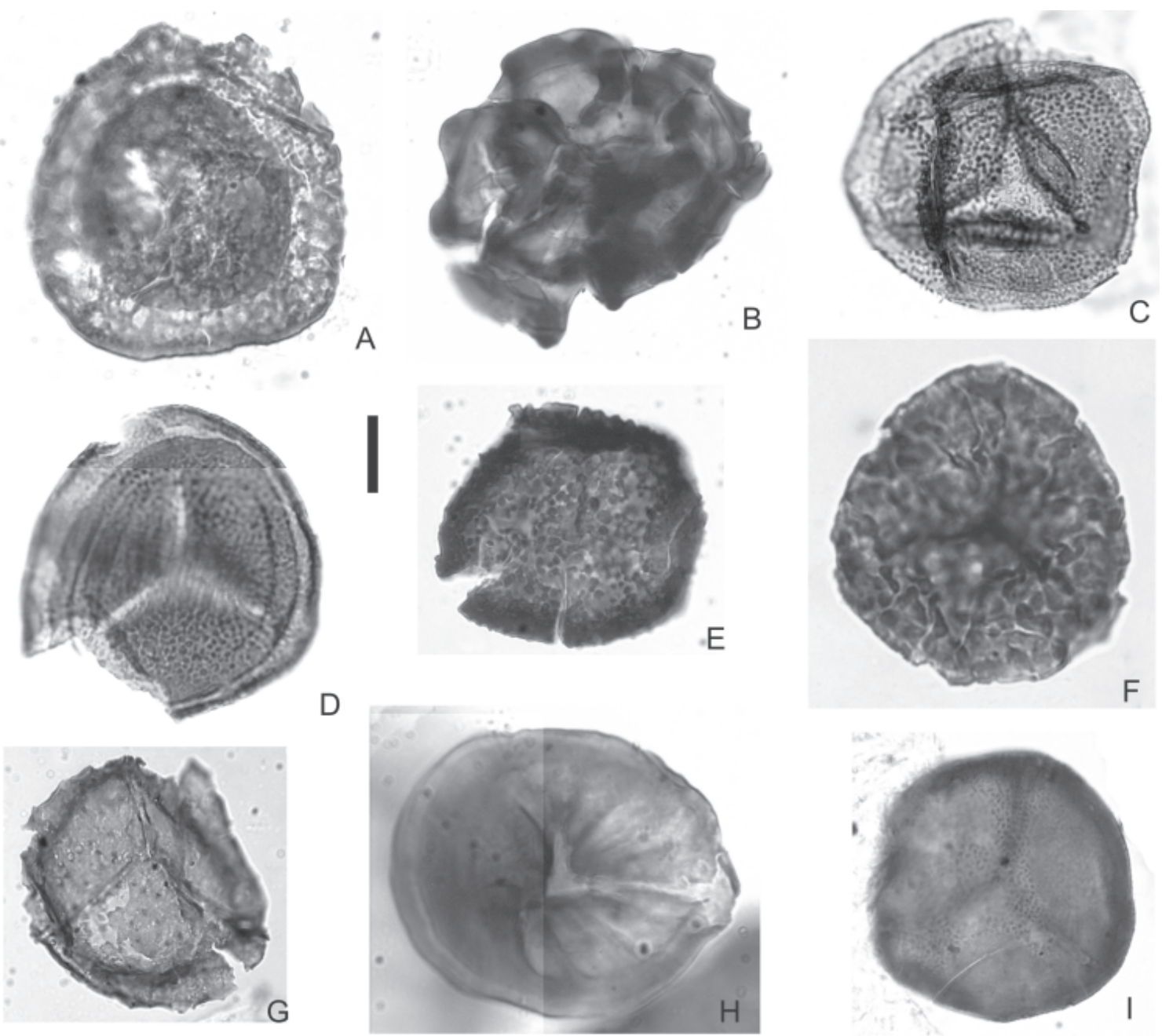

F
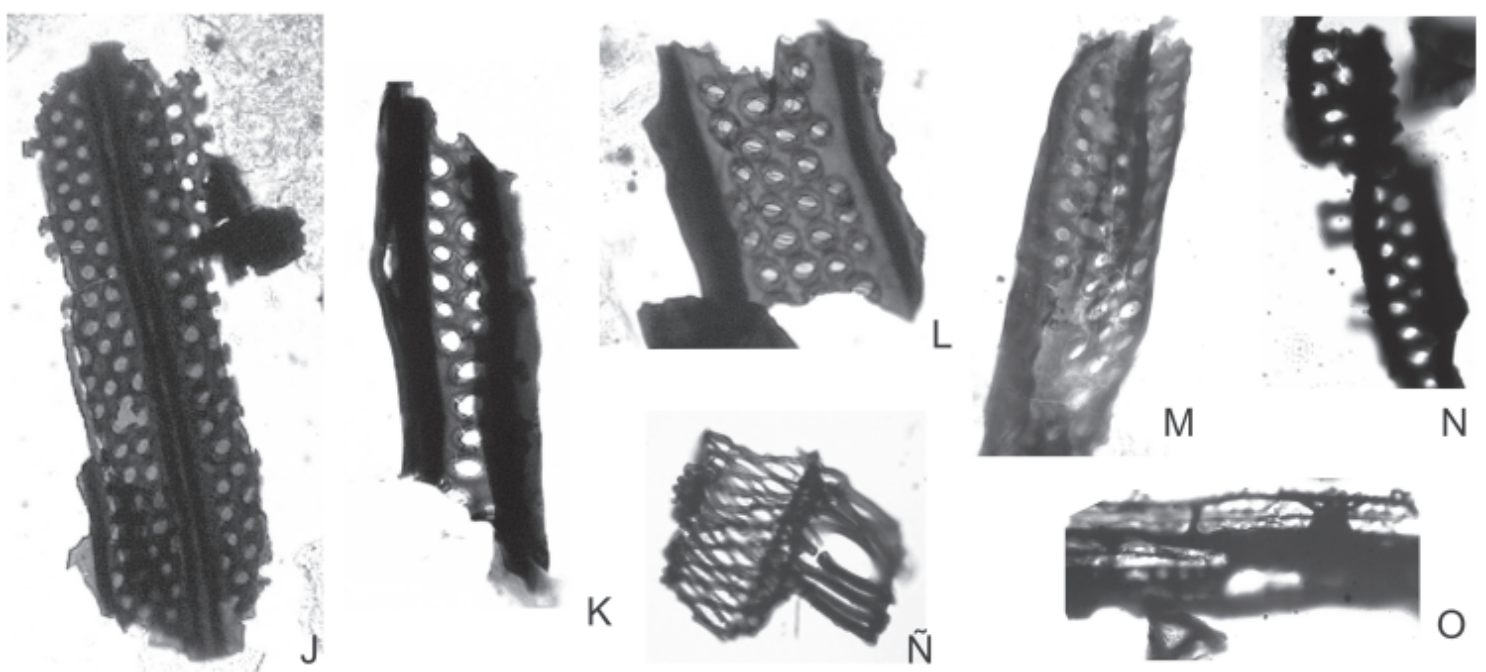


\title{
LÁMINA 6
}

Esporas y microplancton retrabajados presentes en la Formación Tarija.

Escala gráfica: A, C, G, H, N, Q $10 \mu \mathrm{m}, \mathrm{B}, \mathrm{E}, \mathrm{D}, \mathrm{F}, \mathrm{I}-\mathrm{N}, \mathrm{O}, \mathrm{R} 20 \mu \mathrm{m}, \mathrm{P} 15 \mu \mathrm{m}$.

\author{
A Colatisporites decorus (Bharadwaj y Venkatachala) Williams en Neves et al., BAFC-P1 1491(1) K28/2. \\ B, E Ancyrospora spp. B. BAFC-P1 1492(1) V57/3. E. BAFC-Pl 1492(1) Z30/4. \\ C Geminospora spongiata Higgs, Clayton y Keegan. BAFC-Pl 1492(1) L38/4. \\ D Microreticulatisporites parvirugosus Staplin. BAFC-P1 1491(2) N44/1. \\ F $\quad$ Buedingisphaeridium sp. BAFC-P1 1493(1) D46. \\ G Verrucosisporites premnus Richardson. BAFC-P1 1492(1) L33/2. \\ H Origmahapsis pachyderma Colbath. BAFC-P1 1492(1) H43/2. \\ I Maranhites insulatus Burjack y Oliveira. BAFC-P1 1492(1) F27/2. \\ J Striatotheca frequens Burmann 1970. BAFC-P1 1492(1) R26/3. \\ K Maranhites brasiliensis Brito emend. Burjack y Oliveira. BAFC-P1 1492(1) M32/2. \\ L Chomotriletes vedugensis Naumova. BAFC-P1 1480(3) M25/3. \\ M Petrovina connata Oliveira y Burjack. BAFC-P1 1492(1) Z26. \\ N Dictyotidium munificus (Wicander y Wood) Amenábar, di Pasquo, Carrizo y Azcuy. BAFC-Pl 1492(1) \\ C32/1. \\ Ñ, Q Gorgonisphaeridium spp. Ñ. BAFC-P1 1492(1) K35/4. Q. BAFC-P1 1492(1) C32/1. \\ O Amonidium garrasinoi Ottone. BAFC-P1 1492(1) P54/1. \\ P Verhyachium trispininflatum Cramer. BAFC-P1 1492(1) M30. \\ R Quitinozoario. BAFC-P1 1492(1) J31/1.
}


LÁMINA 6

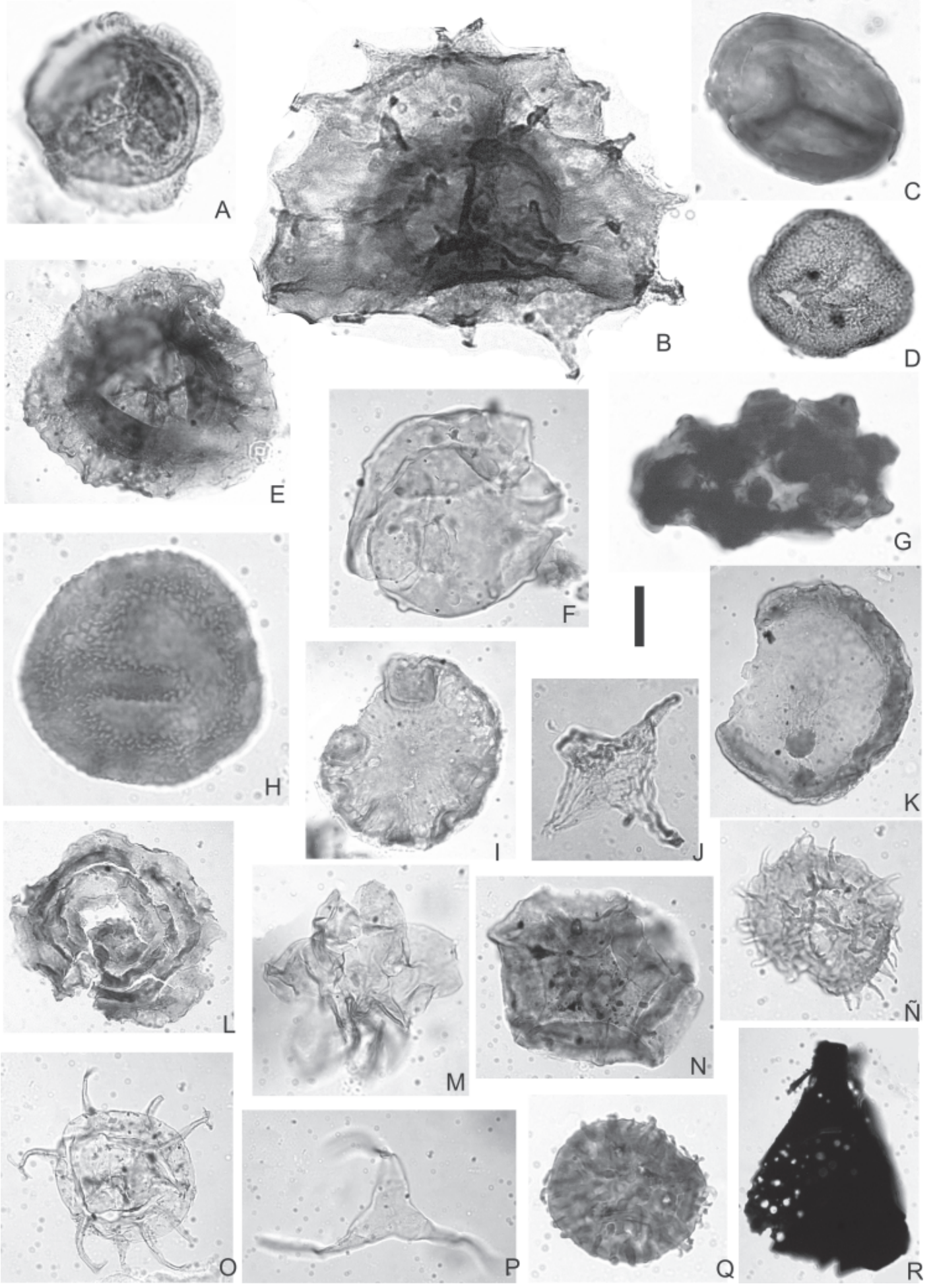

